\title{
Stability and Hopf bifurcation analysis of fractional-order complex-valued neural networks with time delays
}

\author{
R Rakkiyappan ${ }^{1 *}$, K Udhayakumar $^{1}$, G Velmurugan ${ }^{1}$, Jinde $\mathrm{CaO}^{2,3 *}$ and Ahmed Alsaedi ${ }^{3}$
}

"Correspondence:
rakkigru@gmail.com;
jdcao@seu.edu.cn
1Department of Mathematics,
Bharathiar University, Coimbatore,
Tamilnadu 641 046, India
${ }^{2}$ School of Mathematics and
Research Center for Complex
Systems and Network Sciences,
Southeast University, Nanjing,
210096, China
Full list of author information is
available at the end of the article

\begin{abstract}
This paper considers a class of fractional-order complex-valued Hopfield neural networks (CVHNNs) with time delay for analyzing the dynamic behaviors such as local asymptotic stability and Hopf bifurcation. In the case of a neural network with hub and ring structure, the stability of the equilibrium state is investigated by analyzing the eigenvalue of the corresponding characteristic matrix for the hub and ring structured fractional-order time delay models using a Laplace transformation for the Caputo-fractional derivatives. Some sufficient conditions are established to guarantee the uniqueness of the equilibrium point. In addition, conditions for the occurrence of a Hopf bifurcation are also presented. Finally, numerical examples are given to demonstrate the effectiveness of the derived results.
\end{abstract}

Keywords: Hopfield neural networks; fractional-order; time delays; hub structure; ring structure; stability; Hopf bifurcation

\section{Introduction}

The discipline of neural networks, as other fields of science, has a long history of evolution with lots of ups and downs. In 1943 Warren McCulloch and Walter Pitts presented the first model of artificial neurons, named the Threshold Logic Unit (TLU). In the last few decades, the subjective analysis of neural networks (NNs) has received huge attention because of its strong applications in numerous fields such as signal and image processing, associative memories, combinatorial optimization and many others [1-6]. However, such practical applications of NNs are strongly dependent on the qualitative behaviors of NNs. In both biological and physical models, the occurrence of time delays plays an important role. Time delay, which happens usually due to system process and information flow to a particular part of dynamical systems, is unavoidable. Time delays in NNs may cause unexpected dynamical behaviors, like oscillation and poor performance, in networks; see [7-11]. Thus, the analysis on NNs with the effects of time delays has attracted the attention of many researchers and results have been published [12-17]. The stability of neural networks with both leakage delay and a reaction-diffusion term is discussed, and several sufficient conditions were obtained with the help of analysis technique and Lyapunov theory [16]. The problem of fixed-time synchronization of memristive neural networks was studied in [17].

(c) The Author(s) 2017. This article is distributed under the terms of the Creative Commons Attribution 4.0 International License (http://creativecommons.org/licenses/by/4.0/), which permits unrestricted use, distribution, and reproduction in any medium, provided you give appropriate credit to the original author(s) and the source, provide a link to the Creative Commons license, and indicate if changes were made. 
Fractional calculus was introduced many years ago $[8,9,18,19]$, but in recent years the development of fractional calculus was improved step by step in many fields such as engineering mathematics, applied mathematics, and many fields of physics. Thus, recently researchers were strongly attracted towards fractional-order systems compared with the integer-order one because of their great advantage of infinite memory and hereditary properties [3, 19-22]. In the field of neural networks, since the importance of the memory term is extremely high, the incorporation of fractional terms into neural networks leads to a new class of networks called fractional-order NNs [23]. Fractional-order neural networks with parameters such as state vectors, nonlinear activation functions and weight functions in a complex domain $\mathbb{C}^{n}$ are called fractional-order complex-valued neural networks (CVNNs) [3, 7, 24-28]. CVNNs has more remarkable applications in various dynamical systems, such as Lyapunov asymptotic stability, global stability and Hopf bifurcations compared with the real-valued NNs $[8,18,29-32]$. In real-valued NNs, continuously differentiable and bounded sigmoid activation functions are to be chosen, but in the case of a complex domain the activation functions are entire bounded, and they will reduce to a constant in the complex domain according to the Liouville theorem [18]. Therefore in the complex domain choosing the appropriate activation function is a great challenge $[7$, 33].

NNs contain several types of structures such as hub structure, ring structure and so on. In NNs, for appropriate neuronal wiring, it is necessary to have coordinated activation of neuronal assemblies. Hypothetically a few highly linked neurons with long ranging connectivity, the 'hub neurons', would be the most effective manner to organize network-wide synchronicity. Therefore, it is significant to consider the hub structure in neural networks to realize better performance. Moreover, ring architectures can be found in a number of neural structures from neurons in the mind and other sciences. The factual cortical bonding prototype is tremendously sparse. Almost all connections among adjacent cells and long range links became very rare. Thus, ring NNs can be analyzed to gain better understanding of the mechanisms underlying the performance of NNs. Many authors have discussed global stability, Lyapunov stability for the fractional-order NNs, see [2, 14, 27, $29,33-37$, and for finite-time stability results are discussed in [26] and bifurcation of a delayed fractional systems is considered in [5, 38-40].

Stability theory is one of the most important and rapidly developing fields of applied mathematics and mechanics. In the design of a dynamical neural network it is always of interest to study the stability properties of the network. The stability studies in NNs have been developed finding the conditions which ensure that each trajectory of the network converges to an equilibrium point depending on the initial conditions. These completely stable NNs have been used as computing and cognitive machines. Moreover, it is well known that the dynamic behaviors such as periodic phenomenon, bifurcation and chaos are also of great interest. In general, in a dynamical system, if a parameter is allowed to vary, then the behavior of the whole dynamical system may change. The value of the parameter at which these changes occur is known as the bifurcation value and the parameter that is varied is known as the bifurcation parameter. In delay differential equations, Hopf bifurcation occurs in systems of differential equations consisting of two or more equations. Even though there is an extensive literature on bifurcation analysis of some special NNs, most of them deal only with the two-equation models and there were only few papers on the bifurcations of the high-dimensional models [41-46]. However, all above mentioned 
works are on the bifurcation analysis of integer-order NNs. Nevertheless, most results on the integer-order NNs cannot be simply extended to the case of the fractional-order one. To the best of our knowledge, no work on the Hopf bifurcation analysis of fractionalorder CVHNNs with hub structure and ring structure has been proposed in the literature. Therefore, this paper aims at fulfilling such a gap by investigating the stability and Hopf bifurcation of a class of delayed fractional-order CVHNNs with hub structure and ring structure.

Motivated by the above discussion, in this paper we investigate the stability and Hopf bifurcation of the fractional-order CVHNNs with time delays in two types of structures named ring and hub structures. First we consider the fractional-order CVHNNs in the hub structure with time delays. Later the sufficient conditions for stability of the fractional system with respect to the equilibrium point of the system are derived by using some inequality techniques. Further, the bifurcation point of the hub structure with the corresponding critical frequency of the system and for the case similar to the ring structured one are derived. By using the bifurcation point and critical frequency the transversality condition is verified for the point of bifurcation occurring.

The rest of the paper is organized as follows. Necessary basic definitions and a problem description are given in Section 2. In Section 3, the two structures of fractional-order CVHNNs with time delays to investigate the stability and Hopf bifurcation point of the system are presented and two numerical examples are to validate the efficacy of our theoretical findings in Section 4 and lastly a conclusion is discussed in Section 5.

Notations and preliminaries. The authors have derived the results using the following notations in this paper. $\mathbb{R}^{n}$ and $\mathbb{C}^{n}$ are $\mathrm{n}$-dimensional euclidean space and $\mathrm{n}$-dimensional complex domain, respectively. $z(t)$ is a complex variable and is denoted $z(t)=x(t)+i y(t)$, which is in the complex domain. ${ }_{0}^{C} D_{t}^{\alpha} z(t)$ denotes the Caputo-fractional derivative of the complex variable $z(t)$. There are several definitions of fractional-order derivatives and fractional integrals are extensively used, named the Riemann-Liouville derivative and integrals, the Caputo derivative, and Grunwald-Letnikov derivative etc. In this section we give the definitions of the first two.

Definition 1 ([6]) The Riemann-Liouville fractional derivatives $D_{a+}^{\alpha} g$ and $D_{b-}^{\alpha} g$ of order $\alpha \in C([R(\alpha)] \geq 0)$ are defined by

$$
\left(D_{a+}^{\alpha} g\right)(y)=\frac{1}{\Gamma(n-\alpha)}\left(\frac{d}{d y}\right)^{n} \int_{a}^{y} \frac{g(\gamma) d \gamma}{(y-\gamma)^{\alpha-n+1}} \quad(n=[R(\alpha)]+1 ; y>a)
$$

and

$$
\left(D_{b-}^{\alpha} g\right)(y)=\frac{1}{\Gamma(n-\alpha)}\left(\frac{d}{d y}\right)^{n} \int_{y}^{b} \frac{g(\gamma) d \gamma}{(\gamma-y)^{\alpha-n+1}} \quad(n=[R(\alpha)]+1 ; y<b),
$$

where $[R(\alpha)]$ means that integral part of $R(\alpha)$.

Definition 2 ([13]) The Riemann-Liouville fractional integral of order $\alpha>0$ for a continuous function $g: \mathbb{R}^{+} \rightarrow \mathbb{R}$ is defined as

$$
I^{\alpha} g(y)=\frac{1}{\Gamma(\alpha)} \int_{0}^{y}(y-\gamma)^{(\alpha-1)} g(\gamma) d \gamma,
$$


where $I^{\alpha}$ denotes the Riemann-Liouville fractional integral of order $\alpha$ and $\Gamma(\alpha)$ is the Gamma function.

Definition 3 ([47]) The Caputo-fractional derivative of order $\alpha>0$ for a function $g \in$ $\mathbb{C}^{n+1}([0, \infty), \mathbb{R})$ is as follows (the set of all $n+1$ order continuous differentiable functions on $[0, \infty)$ is used):

$$
\begin{aligned}
{ }_{0}^{C} D_{t}^{\alpha} g(t) & =I^{n-\alpha} D^{n} g(t), \\
& =\frac{1}{\Gamma(n-\alpha)} \int_{0}^{t}(t-\gamma)^{(n-\alpha-1)} g^{(n)}(\gamma) d \gamma,
\end{aligned}
$$

where $n$ is the first integer greater than $\alpha$, that is, $n-1<\alpha<n$.

Especially, when $\alpha \in(0,1)$

$$
{ }_{0}^{C} D_{t}^{\alpha} g(t)=\frac{1}{\Gamma(1-\alpha)} \int_{0}^{t}(t-\gamma)^{-\alpha} g^{\prime}(\gamma) d \gamma .
$$

Definition 4 ([13]) The Gamma function is defined as

$$
\Gamma(z)=\int_{0}^{\infty} e^{-t} t^{z-1} d t \quad(\operatorname{Re}(z)>0)
$$

where the $\operatorname{Re}(z)$ is the real part of $z$.

\section{Model description}

In this section we announce the stability analysis of the following fractional-order CVHNNs system with time delays:

$$
{ }_{0}^{C} D_{t}^{\alpha} z_{r}(t)=-c_{r} z_{r}(t)+\sum_{m=1}^{n} a_{r m} f_{m}\left(z_{m}(t)\right)+\sum_{m=1}^{n} b_{r m} f_{m}\left(z_{m}\left(t-\gamma_{r m}\right)\right), \quad r=1,2,3, \ldots, n .
$$

Here, $\alpha \in(0,1), z_{r}$ are the complex state variables, $\left(z(t)=\left(z_{1}(t), z_{2}(t), z_{3}(t), \ldots, z_{n}(t)\right)^{T}\right), c_{r} \in$ $\mathbb{R}^{n \times n}$ are the self-regulating parameters of the neurons, $A=\left(a_{r m}\right)_{n \times n}, B=\left(b_{r m}\right)_{n \times n}$ are the complex inter connection matrices without and with time delays $t$ and $t-\gamma_{r m}$. The $f_{m}$ : $\mathbb{C} \rightarrow \mathbb{C}$ are the complex-valued activation functions for the input-output neurons and the system (1) can be written as

$$
{ }_{0}^{C} D_{t}^{\alpha} Z(t)=-C Z(t)+A F(z(t))+B F(z(t-\gamma)),
$$

where $Z(t)=\left(z_{1}(t), z_{2}(t), z_{3}(t), \ldots, z_{n}(t)\right)^{T}, C=\operatorname{diag}\left(c_{1}, c_{2}, c_{3}, \ldots, c_{n}\right) \in \mathbb{R}^{n \times n}\left(\right.$ for each $\left.c_{r}>0\right)$, $A=\left(a_{r m}\right)_{n \times n} \in \mathbb{C}^{n \times n}, B=\left(b_{r m}\right)_{n \times n} \in \mathbb{C}^{n \times n}$.

The activation functions are given by $F(z(t))=\left(f_{1}\left(z_{1}(t)\right), f_{2}\left(z_{2}(t)\right), f_{3}\left(z_{3}(t)\right), \ldots, f_{n}\left(z_{n}(t)\right)\right)^{T}$, $F(z(t-\gamma))=\left(f_{1}\left(z_{1}\left(t-\gamma_{r 1}\right)\right), f_{2}\left(z_{2}\left(t-\gamma_{r 2}\right)\right), \ldots, f_{n}\left(z_{n}\left(t-\gamma_{r n}\right)\right)\right)^{T}, r=1,2,3, \ldots, n$. We denote $z(t)=x(t)+i y(t), z\left(t_{\gamma}\right)=z(t-\gamma)=x(t-\gamma)+i y(t-\gamma), A=A^{R}+i A^{I}, B=B^{R}+i B^{I}$ and $f(z)=f^{R}(x, y)+i f^{I}(x, y)$ with $x, y: \mathbb{R} \rightarrow \mathbb{R}^{n}, A^{R}, A^{I}, B^{R}, B^{I} \in \mathbb{R}^{n \times n}$; the system (2) can be equivalently written as the following real-valued fractional-order system:

$$
\begin{aligned}
& { }_{0}^{C} D_{t}^{\alpha} x(t)=-C x(t)+A^{R} F^{R}(z(t))+B^{R} F^{R}\left(z\left(t_{\gamma}\right)\right)-A^{I} F^{I}(z(t))-B^{I} F^{I}\left(z\left(t_{\gamma}\right)\right), \\
& { }_{0}^{C} D_{t}^{\alpha} y(t)=-C y(t)+A^{I} F^{R}(z(t))+B^{I} F^{R}\left(z\left(t_{\gamma}\right)\right)+A^{R} F^{I}(z(t))+B^{R} F^{I}\left(z\left(t_{\gamma}\right)\right) .
\end{aligned}
$$


Now, consider $u(t)=(x(t), y(t))^{T} \in \mathbb{R}^{2 n}$,

$$
\tilde{C}=\left(\begin{array}{ll}
C & 0 \\
0 & C
\end{array}\right), \quad \tilde{A}=\left(\begin{array}{cc}
A^{R} & -A^{I} \\
A^{I} & A^{R}
\end{array}\right), \quad \tilde{B}=\left(\begin{array}{cc}
B^{R} & -B^{I} \\
B^{I} & B^{R}
\end{array}\right)
$$

The system (3) and (4) can be written as

$$
{ }_{0}^{C} D_{t}^{\alpha} u(t)=-\tilde{C} u(t)+\tilde{A} \tilde{F}(u(t))+\tilde{B} \tilde{F}\left(u\left(t_{\gamma}\right)\right) .
$$

Let us consider $u^{*}=\left(x^{*}, y^{*}\right)^{T}$ to be an equilibrium state of the system (1). In general, for the linearization of system (5) at the equilibrium point the system can be written as

$$
{ }_{0}^{C} D_{t}^{\alpha} u(t)=-C u(t)+\bar{A} u(t)+\bar{B} u(t-\gamma)
$$

Here $C=\operatorname{diag}\left(c_{1}, c_{2}, c_{3}, \ldots, c_{n}\right), \bar{A}^{R}=\left(\bar{a}_{p q}^{R}\right)_{n \times n}, \bar{A}^{I}=\left(\bar{a}_{p q}^{I}\right)_{n \times n}, \bar{B}^{R}=\left(\bar{B}_{p q}^{R}\right)_{n \times n}$ and $\bar{B}^{I}=$ $\left(\bar{b}_{p q}^{I}\right)_{n \times n}$. Now taking the Laplace transform on both sides of the system (6), we get the Jacobian matrix of the system as

$$
J(s)=\left(\begin{array}{cc}
j_{1} & -j_{2} \\
j_{2} & j_{1}
\end{array}\right)
$$

where

$$
j_{1}=\left(\begin{array}{cccc}
s^{\alpha}+c_{1}-\bar{a}_{11}^{R}-\bar{b}_{11}^{R} e^{-s \gamma_{11}} & -\bar{a}_{12}^{R}-\bar{b}_{12}^{R} e^{-s \gamma_{12}} & \cdots & -\bar{a}_{1 n}^{R}-\bar{b}_{1 n}^{R} e^{-s \gamma_{1 n}} \\
-\bar{a}_{21}^{R}-\bar{b}_{21}^{R} e^{-s \gamma_{21}} & s^{\alpha}+c_{2}-\bar{a}_{22}^{R}-\bar{b}_{22}^{R} e^{-s \gamma_{22}} & \cdots & -\bar{a}_{2 n}^{R}-\bar{b}_{2 n}^{R} e^{-s \gamma_{2 n}} \\
\cdots & \cdots & \ddots & \cdots \\
-\bar{a}_{n 1}^{R}-\bar{b}_{n 1}^{R} e^{-s \gamma_{n 1}} & -\bar{a}_{n 2}^{R}-\bar{b}_{n 2}^{R} e^{-s \gamma_{n 2}} & \cdots & s^{\alpha}+c_{n}-\bar{a}_{n n}^{R}-\bar{b}_{n n}^{R} e^{-s \gamma_{n n}}
\end{array}\right)
$$

and

$$
j_{2}=\left(\begin{array}{cccc}
\bar{a}_{11}^{I}+\bar{b}_{11}^{I} e^{-s \gamma_{11}} & \bar{a}_{12}^{I}+\bar{b}_{12}^{I} e^{-s \gamma_{12}} & \cdots & \bar{a}_{1 n}^{I}+\bar{b}_{1 n}^{I} e^{-s \gamma_{1 n}} \\
\bar{a}_{21}^{I}+\bar{b}_{21}^{I} e^{-s \gamma_{21}} & \bar{a}_{22}^{I}+\bar{b}_{22}^{I} e^{-s \gamma_{22}} & \cdots & \bar{a}_{2 n}^{I}+\bar{b}_{2 n}^{I} e^{-s \gamma_{2 n}} \\
\cdots & \cdots & \ddots & \cdots \\
\bar{a}_{n 1}^{I}+\bar{b}_{n 1}^{I} e^{-s \gamma_{n 1}} & \bar{a}_{n 2}^{I}+\bar{b}_{n 2}^{I} e^{-s \gamma_{n 2}} & \cdots & \bar{a}_{n n}^{I}+\bar{b}_{n n}^{I} e^{-s \gamma_{n n}}
\end{array}\right)
$$

The characteristic matrix of the system (6) is $J(s)$ and the characteristic polynomial is obtained by taking the determinant of $J(s)$. The stability of the equilibrium state $Z^{*}=$ $\left(z_{1}^{*}, z_{2}^{*}, \ldots, z_{n}^{*}\right)$ in the fractional-order system is determined by the eigenvalues of $\operatorname{Det}(J(s))$.

Lemma 1 ([3]) If all the roots of the characteristic equation $\operatorname{Det}(J(s))$ have negative real parts, then the zero equilibrium of system (1) is Lyapunov globally asymptotically stable.

Lemma 2 ([3]) If the order of the system (1) lies between 0 and 1 all the characteristic roots of the matrix $J(s)$ satisfy $|\arg (\lambda)|>\frac{\alpha \pi}{2}$ and the characteristic equation $\operatorname{Det}(J(s))$ has no purely imaginary roots for any $\gamma_{r m}>0, r=m=1,2,3, \ldots, n$, then the zero equilibrium solution of system (1) is Lyapunov globally asymptotically stable. 
To determine our theoretical results we make the following hypothesis:

(H11) The nonlinear activation functions $f_{q}(q=1,2,3, \ldots, n)$ satisfies the Lipschitz condition, that is, there exist constants $F_{k}>0$ such that

$$
\left|f_{q}(x)-f_{q}(y)\right| \leq F_{k}|x-y|, \quad \text { for all } x, y \in \mathbb{R} .
$$

$(\mathbb{H} 2)$ There exist constants $\zeta_{p}(p=1,2,3, \ldots, n)$ such that the following inequality holds:

$$
\zeta_{p} c_{p}>\sum_{k=1}^{n} \zeta_{p}\left(F_{k}\left|a_{p k}\right|+F_{k}\left|b_{p k}\right|\right), \quad p=1,2,3, \ldots, n
$$

Lemma 3 ([38]) If hypotheses $(\mathbb{H 1} 1)$ and $(\mathbb{H} 2)$ are satisfied then there exists a unique equilibrium point for the system (1).

Remark 1 From Lemma 3, we can conveniently discuss the existence and uniqueness of the equilibrium point for neural networks.

\section{Main results}

\subsection{Fractional-order CVHNNs with hub structure and time delays}

In this section, we consider the fractional-order CVHNNs for $n \geq 3$ neurons with hub structure and time delays to investigate the stability and Hopf bifurcation phenomenon,

$$
\left\{\begin{array}{c}
{ }_{0}^{C} D_{t}^{\alpha} z_{1}(t)=-c_{1} z_{1}(t)+\sum_{m=1}^{n} a_{1 m} f_{m}\left(z_{m}(t)\right)+b_{1} f_{1}\left(z_{1}\left(t-\gamma_{1}\right)\right), \\
{ }_{0}^{C} D_{t}^{\alpha} z_{r}(t)=-c_{r} z_{r}(t)+a_{r 1} f_{1}\left(z_{1}(t)\right)+a_{r r} f_{r}\left(z_{r}(t)\right)+b f_{r}\left(z_{r}(t-\gamma)\right), \\
\quad r=2, \ldots, n,
\end{array}\right.
$$

where $\alpha \in \mathbb{R}$ and $0<\alpha<1, c_{1}, c_{r}>0(r=2,3, \ldots, n) . z_{r}(t)$ are complex states. Here, the first neuron (named the central neuron) is the center of the hub, and all the other $r-1$ neurons (named the peripheral neurons) are connected directly only to the central neuron and to themselves. The interconnection matrix of this neural network model (7) is,

$$
\begin{aligned}
A & =\left(\begin{array}{ccccc}
a_{11} & a_{12} & a_{13} & \cdots & a_{1 n} \\
a_{21} & a_{22} & 0 & \cdots & 0 \\
a_{31} & 0 & a_{33} & \cdots & 0 \\
\cdots & \cdots & \cdots & \ddots & \cdots \\
a_{n 1} & 0 & 0 & \cdots & a_{n n}
\end{array}\right) \in \mathbb{C}^{n \times n}, \\
B & =\left(\begin{array}{ccccc}
b_{1} & 0 & 0 & \cdots & 0 \\
0 & b & 0 & \cdots & 0 \\
0 & 0 & b & \cdots & 0 \\
\cdots & \cdots & \cdots & \ddots & \cdots \\
0 & 0 & 0 & \cdots & b
\end{array}\right) \in \mathbb{C}^{n \times n}
\end{aligned}
$$

Let $Z^{*}=\left(z_{1}^{*}, z_{2}^{*}, \ldots, z_{n}^{*}\right)^{T}$ be an equilibrium point of the system (7). The system (7) can be written in the following vector form:

$$
{ }_{0}^{C} D_{t}^{\alpha} Z(t)=-C Z(t)+A F(z(t))+B F\left(z\left(t_{\gamma}\right)\right) \text {, }
$$


with diagonal matrix $C=\left(c_{1}, c_{2}, c_{3}, \ldots, c_{n}\right) \in \mathbb{R}^{n \times n}, A, B \in \mathbb{C}^{n \times n}, F(z(t))=\left(f_{1}\left(z_{1}(t)\right), f_{2}\left(z_{2}(t)\right)\right.$, $\left.f_{3}\left(z_{3}(t)\right), \ldots, f_{n}\left(z_{n}(t)\right)\right)^{T}, F\left(z\left(t_{\gamma}\right)\right)=\left(f_{1}\left(z_{1}\left(t-\gamma_{1}\right)\right), f_{2}\left(z_{2}(t-\gamma)\right), f_{3}\left(z_{3}(t-\gamma)\right), \ldots, f_{n}\left(z_{n}(t-\gamma)\right)\right)^{T}$ for the linearization of system (8) about the equilibrium point of the system (7) and we assume $\gamma_{1}=\gamma$. The system (8) is linearized and the vector form of the system is

$$
{ }_{0}^{C} D_{t}^{\alpha} Z(t)=-C Z(t)+\bar{A} Z(t)+\bar{B} Z(t-\gamma),
$$

where $C=\left\lceil c_{1}, c_{2}, c_{3}, \ldots, c_{n}\right\rfloor$,

$$
\bar{A}=\left(\begin{array}{ccccc}
\bar{a}_{11} & \bar{a}_{12} & \bar{a}_{13} & \cdots & \bar{a}_{1 n} \\
\bar{a}_{21} & \bar{a}_{22} & 0 & \cdots & 0 \\
\bar{a}_{31} & 0 & \bar{a}_{33} & \cdots & 0 \\
\cdots & \ldots & \ldots & \ddots & \ldots \\
\bar{a}_{n 1} & 0 & 0 & \cdots & \bar{a}_{n n}
\end{array}\right), \quad \bar{B}=\left(\begin{array}{ccccc}
\bar{b}_{1} & 0 & 0 & \cdots & 0 \\
0 & \bar{b} & 0 & \cdots & 0 \\
0 & 0 & \bar{b} & \cdots & 0 \\
\ldots & \ldots & \ldots & \ddots & \ldots \\
0 & 0 & 0 & \cdots & \bar{b}
\end{array}\right) .
$$

Taking the Laplace transform on both sides of (9), we have

$$
\begin{aligned}
s^{\alpha} z_{1}(s)-s^{\alpha-1} \psi_{1}(0)= & -c_{1} z_{1}(s)+\bar{a}_{11} z_{1}(s)+\bar{a}_{12} z_{2}(s)+\bar{a}_{13} z_{3}(s)+\cdots+\bar{a}_{1 n} z_{n}(s) \\
& +\bar{b}_{1} e^{-s \gamma}\left(z_{1}(s)+\int_{-\gamma}^{0} e^{-s t} \psi_{1}(t) d t\right), \\
s^{\alpha} z_{2}(s)-s^{\alpha-1} \psi_{2}(0)= & -c_{2} z_{2}(s)+\bar{a}_{21} z_{1}(s)+\bar{a}_{22} z_{2}(s) \\
& +\bar{b} e^{-s \gamma}\left(z_{2}(s)+\int_{-\gamma}^{0} e^{-s t} \psi_{2}(t) d t\right), \\
s^{\alpha} z_{3}(s)-s^{\alpha-1} \psi_{3}(0)= & -c_{3} z_{3}(s)+\bar{a}_{31} z_{1}(s)+\bar{a}_{33} z_{3}(s) \\
& +\bar{b} e^{-s \gamma}\left(z_{3}(s)+\int_{-\gamma}^{0} e^{-s t} \psi_{3}(t) d t\right), \\
\vdots & \\
s^{\alpha} z_{n}(s)-s^{\alpha-1} \psi_{n}(0)= & -c_{n} z_{n}(s)+\bar{a}_{n 1} z_{1}(s)+\bar{a}_{n n} z_{n}(s) \\
& +\bar{b} e^{-s \gamma}\left(z_{n}(s)+\int_{-\gamma}^{0} e^{-s t} \psi_{n}(t) d t\right),
\end{aligned}
$$

where $z_{r}(s)$ is the Laplace transform of $z_{r}(t)$, that is, $z_{r}(s)=L\left(z_{r}(t)\right), r=1,2,3, \ldots, n, \psi_{r}(t)$, $r=1,2,3, \ldots, n, t \in[-\gamma, 0)$, is the initial value of (7). Moreover, the above equation can be rewritten as follows:

$$
J\left(Z^{*}\right) Z(s)=G(s)
$$

where $Z(s)=\left(z_{1}(s), z_{2}(s), z_{3}(s), \ldots, z_{n}(s)\right)^{T}, G(s)=\left(g_{1}(s), g_{2}(s), g_{3}(s), \ldots, g_{n}(s)\right)^{T}, g_{1}(s)=s^{\alpha-1} \times$ $\psi_{1}(0)+\bar{b}_{1} e^{-s \gamma} \int_{-\gamma}^{0} e^{-s t} \psi_{1}(t) d t, g_{2}(s)=s^{\alpha-1} \psi_{2}(0)+\bar{b} e^{-s \gamma} \int_{-\gamma}^{0} e^{-s t} \psi_{2}(t) d t, g_{3}(s)=s^{\alpha-1} \psi_{3}(0)+$ $\bar{b} e^{-s \gamma} \int_{-\gamma}^{0} e^{-s t} \psi_{3}(t) d t, \ldots, g_{n}(s)=s^{\alpha-1} \psi_{n}(0)+\bar{b} e^{-s \gamma} \int_{-\gamma}^{0} e^{-s t} \psi_{n}(t) d t$. 
For convenience, we take

$$
\left\{\begin{array}{l}
c_{1}-\bar{a}_{11}-\bar{b}_{1} e^{-s \gamma}=P, \\
c_{p}-\bar{a}_{p p}-\bar{b} e^{-s \gamma}=Q, \quad p=2,3, \ldots, n .
\end{array}\right.
$$

Then the Jacobian matrix of the system (9) is

$$
J\left(Z^{*}\right)=\left(\begin{array}{ccccc}
s^{\alpha}+P & -\bar{a}_{12} & -\bar{a}_{13} & \cdots & -\bar{a}_{1 n} \\
-\bar{a}_{21} & s^{\alpha}+Q & 0 & \cdots & 0 \\
-\bar{a}_{31} & 0 & s^{\alpha}+Q & \cdots & 0 \\
\cdots & \cdots & \cdots & \ddots & \cdots \\
-\bar{a}_{n 1} & 0 & 0 & \cdots & s^{\alpha}+Q
\end{array}\right) .
$$

It follows that $J\left(Z^{*}\right)$ is an $n \times n$ matrix ( $n \geq 3$ in hub structure). Now we find the determinant of $J\left(Z^{*}\right)$, that is, $\operatorname{Det}\left(J\left(Z^{*}\right)\right)=0$. The characteristic equation $\operatorname{Det}\left(J\left(z^{*}\right)\right)=0$ determines the local stability of the equilibrium solution. The characteristic roots of $J\left(Z^{*}\right)$ satisfy the characteristic equation $\operatorname{Det}\left(J\left(z^{*}\right)\right)=0$.

If $n=3$, the characteristic equation $\operatorname{Det}\left(J\left(Z^{*}\right)\right)=0$ satisfies

$$
\operatorname{Det}\left(J\left(Z^{*}\right)\right)=\left(s^{\alpha}+Q\right)\left(s^{2 \alpha}+(P+Q) s^{\alpha}+P Q-H\right)
$$

If $n=4$, the characteristic equation $\operatorname{Det}\left(J\left(Z^{*}\right)\right)=0$ satisfies

$$
\operatorname{Det}\left(J\left(Z^{*}\right)\right)=\left(s^{\alpha}+Q\right)^{2}\left(s^{2 \alpha}+(P+Q) s^{\alpha}+P Q-H\right)
$$

If $n=5$, the characteristic equation $\operatorname{Det}\left(J\left(Z^{*}\right)\right)=0$ satisfies

$$
\operatorname{Det}\left(J\left(Z^{*}\right)\right)=\left(s^{\alpha}+Q\right)^{3}\left(s^{2 \alpha}+(P+Q) s^{\alpha}+P Q-H\right)
$$

where $P=c_{1}-\bar{a}_{11}-\bar{b}_{1} e^{-s \gamma}, Q=c_{r}-\bar{a}_{r r}-\bar{b} e^{-s \gamma}, H=\sum_{m=2}^{n} a_{1 m} a_{m 1}, r=2,3, \ldots, n$.

The characteristic equation $\operatorname{Det}\left(J\left(Z^{*}\right)\right)=0$ satisfies

$$
\begin{aligned}
\operatorname{Det}\left(J\left(Z^{*}\right)\right)= & \left(s^{\alpha}+c_{r}-\bar{a}_{r r}-\bar{b} e^{-s \gamma}\right)^{n-2}\left(s^{2 \alpha}+\left(c_{1}-\bar{a}_{11}-\bar{b}_{1} e^{-s \gamma}+c_{r}\right.\right. \\
& \left.-\bar{a}_{r r}-\bar{b} e^{-s \gamma}\right) s^{\alpha}+\left(c_{1}-\bar{a}_{11}-\bar{b}_{1} e^{-s \gamma}\right)\left(c_{r}-\bar{a}_{r r}-\bar{b} e^{-s \gamma}\right) \\
& \left.-\sum_{m=2}^{n} a_{1 m} a_{m 1}\right)=0
\end{aligned}
$$

with $P, Q, H \in \mathbb{C}$ defined above depending on the equilibrium $\mathbb{Z}^{*}$ and the system parameters $c_{1}, c_{r}, a_{r m}, b_{1}, b$. Then the characteristic equation (13) will have the form

$$
s^{2 \alpha}+(P+Q) s^{\alpha}+P Q-H=0 .
$$

If the peripheral neurons are identical, then for convenience we take $Q=c_{2}-\bar{a}_{22}-\bar{b} e^{-s \gamma}$. Then (14) can be written as

$s^{2 \alpha}+\left(c_{1}-\bar{a}_{11}-\bar{b}_{1} e^{-s \gamma}+c_{2}-\bar{a}_{22}-\bar{b} e^{-s \gamma}\right) s^{\alpha}+\left(c_{1}-\bar{a}_{11}-\bar{b}_{1} e^{-s \gamma}\right)\left(c_{2}-\bar{a}_{22}-\bar{b} e^{-s \gamma}\right)-H=0$, then 
it can be written in the form of

$$
s^{2 \alpha}+C_{1} s^{\alpha}+C_{2}+\left(C_{3}+C_{4} s^{\alpha}\right) e^{-s \gamma}+C_{5} e^{-2 s \gamma}=0
$$

where $C_{l}(l=1,2, \ldots, 5)$ are defined by $C_{1}=\left(\left(c_{1}+c_{2}\right)-\left(\bar{a}_{11}+\bar{a}_{22}\right)\right), C_{2}=\left(c_{1} c_{2}-c_{1} \bar{a}_{22}-\bar{a}_{11} c_{2}+\right.$ $\left.\bar{a}_{11} \bar{a}_{22}-H\right), C_{3}=\bar{a}_{11} \bar{b}+\bar{a}_{22} \bar{b}_{1}-\bar{b}_{1} c_{2}-\bar{b} c_{1}, C_{4}=-\left(\bar{b}_{1}+\bar{b}\right), C_{5}=\bar{b}_{1} \bar{b}$. For investigating (15), we obtain the following characteristic polynomial:

$$
E_{1}(s)+E_{2}(s) e^{-s \gamma}+E_{3}(s) e^{-2 s \gamma}=0,
$$

where $E_{1}(s)=s^{2 \alpha}+C_{1} s^{\alpha}+C_{2}, E_{2}(s)=C_{3}+C_{4} s^{\alpha}, E_{3}(s)=C_{5}$.

By multiplying $e^{s \gamma}$ on both sides of (16), we get

$$
E_{1}(s) e^{s \gamma}+E_{2}(s)+E_{3}(s) e^{-s \gamma}=0 .
$$

Let $A_{r}$ and $B_{r}$ be the real and imaginary parts of $E_{r}(s)(r=1,2,3)$ and $A_{r}, B_{r}(r=1,2,3)$ are defined as

$$
\begin{aligned}
& A_{1}=\omega^{2 \alpha} \cos \alpha \pi+c_{11} \omega^{\alpha} \cos \frac{\alpha \pi}{2}-c_{12} \omega^{\alpha} \sin \frac{\alpha \pi}{2}+c_{21}, \\
& A_{2}=c_{31} \omega^{\alpha} \cos \frac{\alpha \pi}{2}-c_{32} \omega^{\alpha} \sin \frac{\alpha \pi}{2}+c_{41}, \\
& A_{3}=c_{51}, \quad B_{1}=\omega^{2 \alpha} \sin \alpha \pi+c_{11} \omega^{\alpha} \sin \frac{\alpha \pi}{2}+c_{12} \omega^{\alpha} \sin \frac{\alpha \pi}{2}+c_{22}, \\
& B_{2}=c_{31} \omega^{\alpha} \sin \frac{\alpha \pi}{2}+c_{32} \omega^{\alpha} \cos \frac{\alpha \pi}{2}+c_{42}, \quad B_{3}=c_{52},
\end{aligned}
$$

and

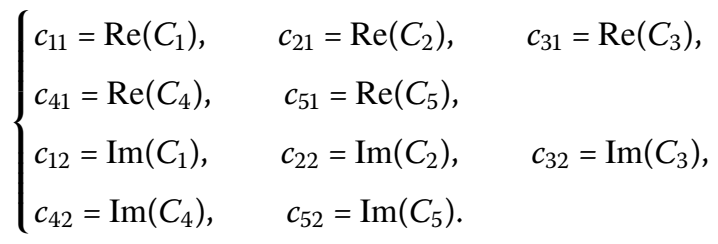

Then by using $A_{r}, B_{r}$ and substituting (18) in (17) we get

$$
\left(A_{1}+i B_{1}\right) e^{s \gamma}+\left(A_{2}+i B_{2}\right)+\left(A_{3}+i B_{3}\right) e^{-s \gamma}=0 .
$$

Further, we will analyze stability and bifurcation properties; for this we consider the real number $\omega>0$, there exists $s=\omega i=\omega\left(\cos \frac{\pi}{2}+i \sin \frac{\pi}{2}\right)$, then we substitute the expression of $s$ into (19), which gives

$$
\begin{aligned}
& \left(A_{1}+i B_{1}\right) e^{i \omega \gamma}+\left(A_{2}+i B_{2}\right)+\left(A_{3}+i B_{3}\right) e^{-i \omega \gamma}=0, \\
& \left(A_{1}+i B_{1}\right)(\cos \omega \gamma+i \sin \omega \gamma)+\left(A_{2}+i B_{2}\right)+\left(A_{3}+i B_{3}\right)(\cos \omega \gamma-i \sin \omega \gamma)=0,
\end{aligned}
$$

then separating the real and imaginary parts of (20) and squaring both sides we get

$$
\begin{aligned}
& \left(A_{1}^{2}+B_{1}^{2}-A_{2}^{2}-B_{2}^{2}-A_{3}^{2}-B_{3}^{2}\right)-2 \cos \omega \gamma\left(A_{2} A_{3}+B_{2} B_{3}\right) \\
& \quad=-2 \sin \omega \gamma\left(A_{3} B_{2}-A_{2} B_{3}\right) .
\end{aligned}
$$


According to $\sin \omega \gamma= \pm \sqrt{1-\cos ^{2} \omega \gamma}$, this leads to following two cases.

Case (a): If $\sin \omega \gamma=\sqrt{1-\cos ^{2} \omega \gamma}$, then it takes the following form:

$$
\begin{aligned}
& \left(A_{1}^{2}+B_{1}^{2}-A_{2}^{2}-B_{2}^{2}-A_{3}^{2}-B_{3}^{2}\right)-2 \cos \omega \gamma\left(A_{2} A_{3}+B_{2} B_{3}\right) \\
& =-2 \sqrt{1-\cos ^{2} \omega \gamma}\left(A_{3} B_{2}-A_{2} B_{3}\right) .
\end{aligned}
$$

It can be concluded that

$$
\cos ^{2} \omega \gamma m_{1}+\cos \omega \gamma m_{2}+m_{3}=0
$$

where

$$
\begin{aligned}
m_{1}= & -4 A_{2}^{2} A_{3}^{2}-4 A_{3}^{2} B_{2}^{2}-4 A_{2}^{2} B_{3}^{2}-4 B_{2}^{2} B_{3}^{2}, \\
m_{2}= & 4 A_{1}^{2} A_{2} A_{3}-4 A_{2}^{3} A_{3}-4 A_{2} A_{3}^{3}+4 A_{2} A_{3} B_{1}^{2}-4 A_{2} A_{3} B_{2}^{2}+4 A_{1}^{2} B_{2} B_{3} \\
& -4 A_{2}^{2} B_{2} B_{3}+4 B_{1}^{2} B_{2} B_{3}-4 B_{2}^{2} B_{3}-4 A_{2} A_{3} B_{3}^{2}-4 B_{2} B_{3}^{3}, \\
m_{3}= & -A_{1}^{4}+2 A_{1}^{2} A_{2}^{2}-A_{2}^{4}+2 A_{1}^{2} A_{3}^{2}-2 A_{2}^{2} A_{3}^{2}-A_{3}^{4}-2 A_{1}^{2} B_{1}^{2}+2 A_{2}^{2} B_{1}^{2}+2 A_{3}^{2} B_{1}^{2}-B_{1}^{4} \\
& +2 A_{1}^{2} B_{2}^{2}-2 A_{2}^{2} B_{2}^{2}+2 A_{3}^{2} B_{2}^{2}+2 B_{1}^{2} B_{2}^{2}-B_{2}^{4}-8 A_{2} A_{3} B_{2} B_{3}+2 A_{1}^{2} B_{3}^{2}+2 A_{1}^{2} B_{3}^{2} \\
& +2 A_{2}^{2} B_{3}^{2}-2 A_{3}^{2} B_{3}^{2}-2 B_{2}^{2} B_{3}^{2}-B_{3}^{4} .
\end{aligned}
$$

As we know quadratic equation (22) has roots. We can obtain the expression of $\cos \omega \gamma$ can be deduced from (22) and is denoted as $f_{1}(\omega)$, that is, $\cos \omega \gamma=f_{1}(\omega)$, Then substituting $\cos \omega \gamma=f_{1}(\omega)$ into equation (21) we get the expression of $\sin \omega \gamma$ and it is represented as $f_{2}(\omega)$. Both $f_{1}(\omega)$ and $f_{2}(\omega)$ are functions with respect to $\omega$ and

$$
\sin \omega \gamma=\frac{2 \cos \omega \gamma\left(A_{2} A_{3}+B_{2} B_{3}\right)-\left(A_{1}^{2}+B_{1}^{2}-A_{2}^{2}-B_{2}^{2}-A_{3}^{2}-B_{3}^{2}\right)}{2\left(A_{3} B_{2}-A_{2} B_{3}\right)}
$$

Moreover, $f_{1}^{2}(\omega)+f_{2}^{2}(\omega)=1$. We obtain

$$
\gamma_{1}^{(k)}=\frac{1}{\omega}\left[\arccos f_{1}(\omega)+2 k \pi\right], \quad k=0,1,2, \ldots
$$

Case (b): If $\sin \omega \gamma=-\sqrt{1-\cos ^{2} \omega \gamma}$, similar to Case (a), we can obtain $\cos \omega \gamma=g_{1}(\omega)$ and $\sin \omega \gamma=g_{2}(\omega)$, where $g_{1}(\omega), g_{2}(\omega)$ is a function with respect to $\omega$. Here $\omega$ is the root of the equation $g_{1}^{2}(\omega)+g_{2}^{2}(\omega)=1$. If we take $g_{1}(\omega)=\cos \omega \gamma$, we have

$$
\gamma_{2}^{(k)}=\frac{1}{\omega}\left[\arccos g_{1}(\omega)+2 k \pi\right], \quad k=0,1,2, \ldots
$$

We will take that $f_{1}^{2}(\omega)+f_{2}^{2}(\omega)=1$ or $g_{1}^{2}(\omega)+g_{2}^{2}(\omega)=1$, having at least one positive real root, thus the bifurcation point is defined as

$$
\gamma_{0}=\min \left\{\gamma_{1}^{(k)}, \gamma_{2}^{(k)}\right\}, \quad k=0,1,2, \ldots,
$$

where $\gamma_{1}^{(k)}, \gamma_{2}^{(k)}$ are defined in (23) and (24). 
Now, we examine the stability of system (7) for $\gamma=0$, the characteristic equation (15) becomes

$$
f(\lambda)=\lambda^{2}+C_{1} \lambda+C_{2}+C_{3} \lambda+C_{4}+C_{5}=0
$$

Equation (26) can be converted to

$$
\lambda^{2}+D_{1} \lambda+D_{2}=0
$$

where

$$
D_{1}=C_{1}+C_{3}, \quad D_{2}=C_{2}+C_{4}+C_{5} .
$$

It is defined as

$$
\Psi_{1}=D_{1}, \quad \Psi_{2}=\left|\begin{array}{cc}
D_{1} & 1 \\
D_{3} & D_{2}
\end{array}\right|, \quad \Psi_{3}=\left|\begin{array}{ccc}
D_{1} & 1 & 0 \\
D_{3} & D_{2} & D_{1} \\
0 & D_{4} & D_{3}
\end{array}\right|, \quad \ldots, \quad \Psi_{n}=\Psi_{n-1} D_{n},
$$

from the above we make the following hypotheses:

$(\mathbb{H} 3) \quad \Psi_{i}>0(i=1,2)$.

Lemma 4 If $\Psi_{1}>0$ and $\Psi_{2}>0$ holds, then the zero equilibrium point of the fractionalorder system (7) is asymptotically stable when $\gamma=0$.

Remark 2 The conditions $\Psi_{1}>0$ and $\Psi_{2}>0$ are sufficient condition for Lemma 4 . If the conditions are retrieved by another method which entails that all the roots of equation (13) satisfy $|\arg (\lambda)|>\frac{\alpha \pi}{2}$ then Lemma 4 may still hold.

In order to achieve the transversality condition for the occurrence for Hopf bifurcation, the following further hypothesis is needed:

$\left.(\mathbb{H} 4) \operatorname{Re}\left[\frac{d s}{d \gamma}\right]\right|_{\left(\gamma=\gamma_{0}, \omega=\omega_{0}\right)} \neq 0$, where $\gamma_{0}$ and $\omega_{0}$ are bifurcation point and critical frequency, respectively.

By using the implicit function theorem and taking the derivative of $s$ with respect to $\gamma$ in (16), then

$$
\frac{d s}{d \gamma}=\frac{X(s)}{Y(s)} .
$$

Here

$$
\begin{aligned}
& X(s)=s\left\{\left(C_{3} s^{\alpha}+C_{4}\right) e^{-s \gamma}+2 C_{5} e^{-2 s \gamma}\right\}, \\
& Y(s)=\left\{2 \alpha s^{2 \alpha-1}+C_{1} \alpha s^{\alpha-1}+\left[\alpha C_{3} s^{\alpha-1}-\gamma\left(C_{3} s^{\alpha}+C_{4}\right)\right] e^{-s \gamma}-2 \gamma C_{5} e^{-2 s \gamma}\right\} .
\end{aligned}
$$

It can be deduced from (28) that

$$
\frac{d s}{d \gamma}=\frac{X_{1} Y_{1}+X_{2} Y_{2}+i\left(X_{2} Y_{1}-Y_{2} X_{1}\right)}{Y_{1}^{2}+Y_{2}^{2}},
$$


then we take

$$
\left.\operatorname{Re}\left[\frac{d s}{d \gamma}\right]\right|_{\left(\gamma=\gamma_{0}, \omega=\omega_{0}\right)}=\frac{X_{1} Y_{1}+X_{2} Y_{2}}{Y_{1}^{2}+Y_{2}^{2}}
$$

where $X_{1}, X_{2}$ are the real and imaginary parts of $X(s)$ and $Y_{1}, Y_{2}$ are the real and imaginary parts of $Y(s)$, which are defined as

$$
\begin{aligned}
& X_{1}=\omega_{0} {\left[\left(C_{3} \omega_{0}^{\alpha} \cos \frac{\alpha \pi}{2}+C_{4}\right) \sin \omega_{0} \gamma_{0}-C_{3} \omega_{0}^{\alpha} \sin \frac{\alpha \pi}{2} \cos \omega_{0} \gamma_{0}+2 C_{5} \sin 2 \omega_{0} \gamma_{0}\right], } \\
& X_{2}=\omega_{0}\left[\left(C_{3} \omega_{0}^{\alpha} \cos \frac{\alpha \pi}{2}+C_{4}\right) \cos \omega_{0} \gamma_{0}+C_{3} \omega_{0}^{\alpha} \sin \frac{\alpha \pi}{2} \sin \omega_{0} \gamma_{0}+2 C_{5} \cos 2 \omega_{0} \gamma_{0}\right], \\
& Y_{1}=\left[\alpha C_{3} \omega_{0}^{\alpha-1} \cos \frac{(\alpha-1) \pi}{2}-\gamma_{0} C_{3} \omega_{0}^{\alpha} \cos \frac{\alpha \pi}{2}+C_{4}\right] \cos \omega_{0} \gamma_{0} \\
&+\left[\alpha C_{3} \omega_{0}^{\alpha-1} \sin \frac{(\alpha-1) \pi}{2}-\gamma_{0} C_{3} \omega_{0}^{\alpha} \sin \frac{\alpha \pi}{2}\right] \sin \omega_{0} \gamma_{0}-2 \gamma_{0} C_{5} \cos 2 \omega_{0} \gamma_{0} \\
&+2 \alpha \omega_{0}^{2 \alpha-1} \cos \frac{(2 \alpha-1) \pi}{2}+C_{1} \alpha \omega^{\alpha-1} \cos \frac{(\alpha-1) \pi}{2}, \\
& Y_{2}=-\left[\alpha C_{3} \omega_{0}^{\alpha-1} \cos \frac{(\alpha-1) \pi}{2}-\gamma_{0} C_{3} \omega_{0}^{\alpha} \cos \frac{\alpha \pi}{2}+C_{4}\right] \sin \omega_{0} \gamma_{0} \\
&+\left[\alpha C_{3} \omega_{0}^{\alpha-1} \sin \frac{(\alpha-1) \pi}{2}-\gamma_{0} C_{3} \omega_{0}^{\alpha} \sin \frac{\alpha \pi}{2}\right] \cos \omega_{0} \gamma_{0}+2 \gamma_{0} C_{5} \sin 2 \omega_{0} \gamma_{0} \\
&+2 \alpha \omega_{0}^{2 \alpha-1} \sin \frac{(2 \alpha-1) \pi}{2}+C_{1} \alpha \omega^{\alpha-1} \sin \frac{(\alpha-1) \pi}{2} .
\end{aligned}
$$

From the above investigation we can state the following theorem.

Theorem 1 Assume that ( $\mathbb{H 1} 1)-(\mathbb{H} 4)$ are satisfied for system (7), the following results hold:

(1) The zero equilibrium point is asymptotically stable for $\gamma \in\left[0, \gamma_{0}\right)$.

(2) The system (7) exhibits a Hopf bifurcation at the origin when $\gamma=\gamma_{0}$, that is, the system (7) has a branch of periodic solutions bifurcating from the zero equilibrium point near $\gamma=\gamma_{0}$.

Remark 3 The results on stability and Hopf bifurcation of fractional-order CVHNNs with hub structure and time delays have not been attained before. The determined conditions on bifurcation are very straightforward, detailed and impressive and simple to be verified in the present work by applying Hopf bifurcation theory. Our work is to develop the study of the theory of nonlinear dynamics.

\subsection{Fractional-order CVHNNs with ring structure and time delays}

In this section we consider the fractional-order CVHNNs with ring structured network and time delay as

$$
\left\{\begin{aligned}
{ }_{0}^{c} D_{t}^{\alpha} z_{1}(t)= & -c_{1} z_{1}(t)+a_{11} f_{1}\left(z_{1}(t)\right)+a_{12} f_{2}\left(z_{2}(t)\right)+b_{11} f_{1}\left(z_{1}(t-\gamma)\right) \\
& +b_{12} f_{2}\left(z_{2}(t-\gamma)\right) \\
{ }_{0}^{c} D_{t}^{\alpha} z_{2}(t)= & -c_{2} z_{2}(t)+a_{22} f_{2}\left(z_{2}(t)\right)+a_{21} f_{1}\left(z_{1}(t)\right)+b_{11} f_{2}\left(z_{2}(t-\gamma)\right) \\
& +b_{21} f_{1}\left(z_{1}(t-\gamma)\right)
\end{aligned}\right.
$$


where the order $\alpha \in(0,1), z_{p}(t)(p=1,2)$ are complex states, $c_{r}>0(r=1,2)$ are real values, $\gamma$ is the time delay, $f$ is the nonlinear activation function. A neural network with the ring connectivity structure is in (30), in which every neuron in the network is connected only to its closest neurons. It is clear that the $Z^{*}$ is an equilibrium point of (30). We generate the following hypothesis on the nonlinear activation functions to the system in (30):

(A1) $f_{p} \in \mathbb{C}^{1}(\mathbb{R}, \mathbb{R}), f_{p}(0)=0$,

we may consider steady state $Z^{*}$ with equal complex scalar components $Z_{1}^{*}=Z_{2}^{*}=Z^{*}$.

Obviously, from $(\mathbb{A} 1) Z^{*}=0$ is a solution of this equation as the trivial solution is a steady state of (30), then

$$
\left\{\begin{array}{l}
-c_{1} z_{1}^{*}+a_{11} f_{1}\left(z_{1}^{*}\right)+a_{12} f_{2}\left(z_{2}^{*}\right)+b_{11} f_{1}\left(z_{1}^{*}\right)+b_{12} f_{2}\left(z_{2}^{*}\right)=0 \\
-c_{2} z_{2}^{*}+a_{21} f_{1}\left(z_{1}^{*}\right)+a_{22} f_{2}\left(z_{2}^{*}\right)+b_{21} f_{1}\left(z_{1}^{*}\right)+b_{22} f_{2}\left(z_{2}^{*}\right)=0
\end{array}\right.
$$

Linearization of the above system with respect to the equilibrium state of the system (30) leads to

$$
\begin{aligned}
{ }_{0}^{c} D_{t}^{\alpha} z_{1}(t)= & -c_{1} z_{1}(t)+a_{11}\left[f_{1}\left(z_{1}^{*}\right)+\frac{\partial f_{1}}{\partial z_{1}}\left(z_{1}^{*}\right)\left(z_{1}-z_{1}^{*}\right)\right]+a_{12}\left[f_{2}\left(z_{2}^{*}\right)\right. \\
& \left.+\frac{\partial f_{2}}{\partial z_{2}}\left(z_{2}^{*}\right)\left(z_{2}-z_{2}^{*}\right)\right]+b_{11}\left[f_{1}\left(z_{1}^{*}\right)+\frac{\partial f_{1}}{\partial z_{1}}\left(z_{1}^{*}\right)\left(z_{1}(t-\gamma)-z_{1}^{*}\right)\right] \\
& +b_{12}\left[f_{2}\left(z_{2}^{*}\right)+\frac{\partial f_{2}}{\partial z_{2}}\left(z_{2}^{*}\right)\left(z_{2}(t-\gamma)-z_{2}^{*}\right)\right], \\
{ }_{0}^{c} D_{t}^{\alpha} z_{2}(t)= & -c_{2} z_{2}(t)+a_{22}\left[f_{2}\left(z_{2}^{*}\right)+\frac{\partial f_{2}}{\partial z_{2}}\left(z_{2}^{*}\right)\left(z_{2}-z_{2}^{*}\right)\right]+a_{21}\left[f_{1}\left(z_{1}^{*}\right)\right. \\
& \left.+\frac{\partial f_{1}}{\partial z_{1}}\left(z_{1}^{*}\right)\left(z_{1}-z_{1}^{*}\right)\right]+b_{22}\left[f_{2}\left(z_{2}^{*}\right)+\frac{\partial f_{2}}{\partial z_{2}}\left(z_{2}^{*}\right)\left(z_{2}(t-\gamma)-z_{2}^{*}\right)\right] \\
& +b_{21}\left[f_{1}\left(z_{1}^{*}\right)+\frac{\partial f_{1}}{\partial z_{1}}\left(z_{1}^{*}\right)\left(z_{1}(t-\gamma)-z_{1}^{*}\right)\right] .
\end{aligned}
$$

Then it can be written in the following form:

$$
\begin{aligned}
& { }_{0}^{c} D_{t}^{\alpha} z_{1}(t)=-c_{1} z_{1}(t)+\bar{a}_{11} z_{1}+\bar{a}_{12} z_{2}+\bar{b}_{11} z_{1}(t-\gamma)+\bar{b}_{12} z_{2}(t-\gamma), \\
& { }_{0}^{c} D_{t}^{\alpha} z_{2}(t)=-c_{2} z_{2}(t)+\bar{a}_{22} z_{2}+\bar{a}_{21} z_{1}+\bar{b}_{22} z_{2}(t-\gamma)+\bar{b}_{21} z_{1}(t-\gamma),
\end{aligned}
$$

where $\bar{a}_{r m}=a_{r m} f^{\prime}(0)$ and $\bar{b}_{r m}=b_{r m} f^{\prime}(0), r, m=1,2$.

The system parameters $a_{r m}, b_{r m}(r, m=1,2)$ are connection weights of the neural network and we have the complex domain. We take the Laplace transform on both sides of (32) and (33),

$$
\begin{aligned}
s^{\alpha} Z_{1}(s)-s^{\alpha-1} \psi_{1}(0)= & -c_{1} Z_{1}(s)+\bar{a}_{11} Z_{1}(s)+\bar{a}_{12} Z_{2}(s)+\bar{b}_{11} e^{-s \gamma}\left(Z_{1}(s)+\int_{-\gamma}^{0} e^{-s t} \psi_{1}(t) d t\right) \\
& +\bar{b}_{12} e^{-s \gamma}\left(Z_{2}(s)+\int_{-\gamma}^{0} e^{-s t} \psi_{2}(t) d t\right),
\end{aligned}
$$




$$
\begin{aligned}
& s^{\alpha} Z_{2}(s)-s^{\alpha-1} \psi_{2}(0) \\
& =-c_{2} Z_{2}(s)+\bar{a}_{21} Z_{1}(s)+\bar{a}_{22} Z_{2}(s)+\bar{b}_{21} e^{-s \gamma}\left(Z_{1}(s)+\int_{-\gamma}^{0} e^{-s t} \psi_{1}(t) d t\right) \\
& \quad+\bar{b}_{22} e^{-s \gamma}\left(Z_{2}(s)+\int_{-\gamma}^{0} e^{-s t} \psi_{2}(t) d t\right),
\end{aligned}
$$

where $Z_{1}(s), Z_{2}(s)$ are the Laplace transforms of $z_{1}(t), z_{2}(t)$, that is, $L\left[z_{p}(t)\right]=Z_{p}(s), p=1,2$, and $\psi_{1}(t), \psi_{2}(t)$, are the initial values of the (30). Then it can be written as

$$
\left\{\begin{array}{l}
s^{\alpha} Z_{1}(s)+c_{1} Z_{1}(s)-\bar{a}_{11} Z_{1}(s)-\bar{a}_{12} Z_{2}(s)-\bar{b}_{11} e^{-s \gamma} Z_{1}(s)-\bar{b}_{12} e^{-s \gamma} Z_{2}(s)=L_{1} \\
s^{\alpha} Z_{2}(s)+c_{2} Z_{2}(s)-\bar{a}_{21} Z_{1}(s)-\bar{a}_{22} Z_{2}(s)-\bar{b}_{21} e^{-s \gamma} Z_{1}(s)-\bar{b}_{22} e^{-s \gamma} Z_{2}(s)=L_{2}
\end{array}\right.
$$

where

$$
\begin{aligned}
& L_{1}=s^{\alpha-1} \psi_{1}(0)+\bar{b}_{11} e^{-s \gamma} \int_{-\gamma}^{0} e^{-s t} \psi_{1}(t) d t+\bar{b}_{12} e^{-s \gamma} \int_{-\gamma}^{0} e^{-s t} \psi_{2}(t) d t, \\
& L_{2}=s^{\alpha-1} \psi_{2}(0)+\bar{b}_{21} e^{-s \gamma} \int_{-\gamma}^{0} e^{-s t} \psi_{1}(t) d t+\bar{b}_{22} e^{-s \gamma} \int_{-\gamma}^{0} e^{-s t} \psi_{2}(t) d t .
\end{aligned}
$$

We will study the behavior of dynamical system by using two feasible equilibria. The characteristic equation for system (34) is

$$
\operatorname{Det}\left(\begin{array}{cc}
s^{\alpha}-\left(-c_{1}+\bar{a}_{11}+\bar{b}_{11} e^{-s \gamma}\right) & -\bar{a}_{12}-\bar{b}_{12} e^{-s \gamma} \\
-\bar{a}_{21}-\bar{b}_{21} e^{-s \gamma} & s^{\alpha}-\left(-c_{2}+\bar{a}_{22}+\bar{b}_{22} e^{-s \gamma}\right)
\end{array}\right)=0 .
$$

By (35), we can obtain the characteristic equation

$$
E_{1}(s)+E_{2}(s) e^{-s \gamma}+E_{3}(s) e^{-2 s \gamma}=0
$$

where $E_{1}(s)=s^{2 \alpha}+C_{1} s^{\alpha}+C_{2}, E_{2}(s)=C_{3} s^{\alpha}+C_{4}, E_{3}(s)=C_{5}$.

The coefficients are defined by $C_{1}=-\bar{a}_{11}-\bar{a}_{22}+c_{1}+c_{2}, C_{2}=-\bar{a}_{12} \bar{a}_{21}+\bar{a}_{11} \bar{a}_{22}-2 \overline{2} c_{1}-$ $\bar{a}_{11} c_{2}+c_{1} c_{2}, C_{3}=-\bar{b}_{11}-\bar{b}_{22}, C_{4}=\bar{a}_{11} \bar{b}_{22}-\bar{b}_{22} c_{1}-\bar{b}_{11} c_{2}+\bar{a}_{22} \overline{1}-\bar{a}_{21} \bar{b}_{12}-\bar{a}_{12} \bar{b}_{21}, C_{5}=-\bar{b}_{12} \bar{b}_{21}+$ $\bar{b}_{11} \bar{b}_{22}$ and

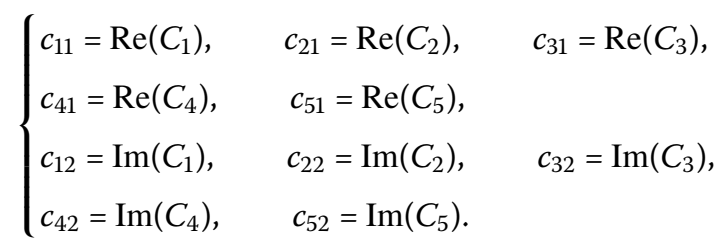

The characteristic equation (36) determines the local stability of the equilibrium solution; the system (30) is stable iff all the eigenvalues of (35), having the solutions of

$$
E_{1}(s)+E_{2}(s) e^{-s \gamma}+E_{3}(s) e^{-2 s \gamma}=0
$$

have negative real parts. It is well known that the equilibrium state $Z^{*}$ is stable if all roots of (36) have negative real parts, and unstable if one root has a positive real part. Multiplying 
$e^{s \gamma}$ on both sides of (38), it is obvious that

$$
E_{1}(s) e^{s \gamma}+E_{2}(s)+E_{3}(s) e^{-2 s \gamma}=0 .
$$

If $s=i \omega=\omega\left(\cos \frac{\pi}{2}+i \sin \frac{\pi}{2}\right), \omega>0$ is a root of (39) iff $\omega$ satisfies the following equations, we obtain

$$
E_{1}(s) e^{i \omega \gamma}+E_{2}(s)+E_{3}(s) e^{-i 2 \omega \gamma}=0
$$

$A_{r}, B_{r}(r=1,2,3)$ are the real and imaginary parts of $E_{r}(s)(r=1,2,3)$, respectively. Then (40) can be written as follows:

$$
\begin{aligned}
& \left(A_{1}+i B_{1}\right) e^{i \omega \gamma}+\left(A_{2}+i B_{2}\right)+\left(A_{3}+i B_{3}\right) e^{-i \omega \omega \gamma}=0, \\
& \left(A_{1}+i B_{1}\right)(\cos \omega \gamma+i \sin \omega \gamma)+\left(A_{2}+i B_{2}\right)+\left(A_{3}+i B_{3}\right)(\cos \omega \gamma-i \sin \omega \gamma)=0 .
\end{aligned}
$$

Separating the real and imaginary parts of the above leads to

$$
\left\{\begin{array}{l}
A_{1} \cos \omega \gamma-B_{1} \sin \omega \gamma=-\left(A_{3} \cos \omega \gamma+B_{3} \sin \omega \gamma+A_{2}\right), \\
A_{1} \sin \omega \gamma+B_{1} \cos \omega \gamma=-\left(B_{3} \cos \omega \gamma-A_{3} \sin \omega \gamma+B_{2}\right),
\end{array}\right.
$$

where

$$
\begin{aligned}
& A_{1}=\omega^{2 \alpha} \cos \alpha \pi+c_{11} \omega^{\alpha} \cos \frac{\alpha \pi}{2}-c_{12} \omega^{\alpha} \sin \frac{\alpha \pi}{2}+c_{21}, \\
& A_{2}=c_{31} \omega^{\alpha} \cos \frac{\alpha \pi}{2}-c_{32} \omega^{\alpha} \sin \frac{\alpha \pi}{2}+c_{41}, \quad A_{3}=c_{51} . \\
& B_{1}=\omega^{2 \alpha} \sin \alpha \pi+c_{11} \omega^{\alpha} \sin \frac{\alpha \pi}{2}+c_{12} \omega^{\alpha} \sin \frac{\alpha \pi}{2}+c_{22}, \\
& B_{2}=c_{31} \omega^{\alpha} \sin \frac{\alpha \pi}{2}+c_{32} \omega^{\alpha} \cos \frac{\alpha \pi}{2}+c_{42}, \quad B_{3}=c_{52} .
\end{aligned}
$$

Accordingly $\sin ^{2} \omega \gamma+\cos ^{2} \omega \gamma=1$ leads to the following two cases.

Case (a): If we take $\sin \omega \gamma=\sqrt{1-\cos ^{2} \omega \gamma}$, then we have

$$
\begin{aligned}
& \left(A_{1}^{2}+B_{1}^{2}-A_{2}^{2}-B_{2}^{2}-A_{3}^{2}-B_{3}^{2}\right)-2 \cos \omega \gamma\left(A_{2} A_{3}+B_{2} B_{3}\right) \\
& \quad=-2 \sqrt{1-\cos ^{2} \omega \gamma}\left(A_{3} B_{2}-A_{2} B_{3}\right) .
\end{aligned}
$$

It can be concluded from that

$$
\cos ^{2} \omega \gamma m_{1}+\cos \omega \gamma m_{2}+m_{3}=0
$$

where

$$
\begin{aligned}
m_{1}= & -4 A_{2}^{2} A_{3}^{2}-4 A_{3}^{2} B_{2}^{2}-4 A_{2}^{2} B_{3}^{2}-4 B_{2}^{2} B_{3}^{2}, \\
m_{2}= & 4 A_{1}^{2} A_{2} A_{3}-4 A_{2}^{3} A_{3}-4 A_{2} A_{3}^{3}+4 A_{2} A_{3} B_{1}^{2}-4 A_{2} A_{3} B_{2}^{2}+4 A_{1}^{2} B_{2} B_{3} \\
& -4 A_{2}^{2} B_{2} B_{3}+4 B_{1}^{2} B_{2} B_{3}-4 B_{2}^{2} B_{3}-4 A_{2} A_{3} B_{3}^{2}-4 B_{2} B_{3}^{3},
\end{aligned}
$$



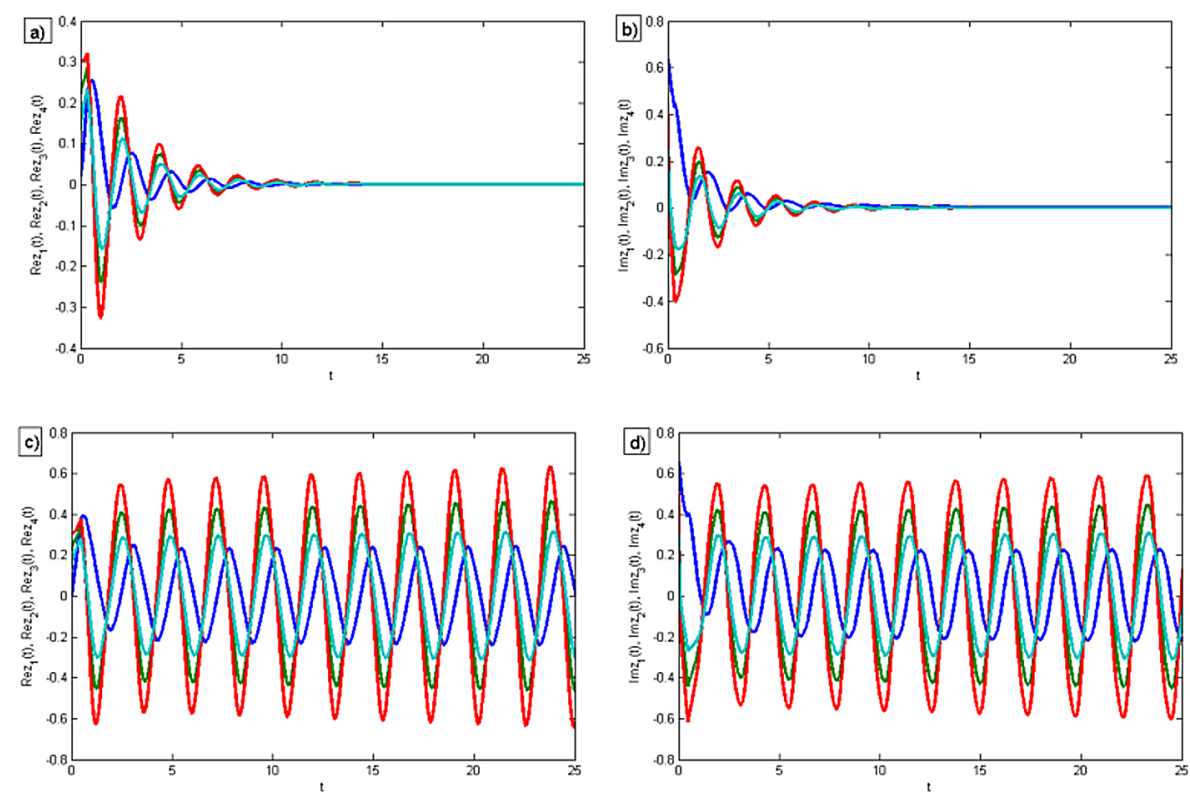

Figure 1 State trajectories of the real and imaginary parts of the fractional-order CVHNN with hub structure (50). $\alpha=0.9$ for $\gamma_{0}=0.32$ in (a) and (b) and $\gamma=0.45>\gamma_{0}=0.32$ in (c) and (d).

$$
\begin{aligned}
m_{3}= & -A_{1}^{4}+2 A_{1}^{2} A_{2}^{2}-A_{2}^{4}+2 A_{1}^{2} A_{3}^{2}-2 A_{2}^{2} A_{3}^{2}-A_{3}^{4}-2 A_{1}^{2} B_{1}^{2}+2 A_{2}^{2} B_{1}^{2}+2 A_{3}^{2} B_{1}^{2}-B_{1}^{4} \\
& +2 A_{1}^{2} B_{2}^{2}-2 A_{2}^{2} B_{2}^{2}+2 A_{3}^{2} B_{2}^{2}+2 B_{1}^{2} B_{2}^{2}-B_{2}^{4}-8 A_{2} A_{3} B_{2} B_{3}+2 A_{1}^{2} B_{3}^{2}+2 A_{1}^{2} B_{3}^{2} \\
& +2 A_{2}^{2} B_{3}^{2}-2 A_{3}^{2} B_{3}^{2}-2 B_{2}^{2} B_{3}^{2}-B_{3}^{4} .
\end{aligned}
$$

From this we can obtain the interpretation of the $\cos \omega \gamma$ and denote $\cos \omega \gamma=d_{1}(\omega)$. Using the expression of $\cos \omega \gamma$ in (41) then we get $\sin \omega \gamma$ and it is denoted $\sin \omega \gamma=d_{2}(\omega)$, where $d_{1}(\omega)$ and $d_{2}(\omega)$ is functions with respect to $\omega$.

We know that $\sin ^{2} \omega \gamma+\cos ^{2} \omega \gamma=1$, from this identity we obtain $d_{1}^{2}(\omega)+d_{2}^{2}(\omega)=1$.

Hence, we get

$$
\gamma_{1}^{(j)}=\frac{1}{\omega}\left[\arccos d_{1}(\omega)+2 j \pi\right], \quad j=0,1,2, \ldots
$$

Case (b): Now consider $\sin \omega \gamma=-\sqrt{1-\cos ^{2} \omega \gamma}$, in the same way as Case (a), we can obtain $\cos \omega \gamma=h_{1}(\omega)$, where $h_{1}(\omega), h_{2}(\omega)$ is a function with respect to $\omega$. It is the roots of the polynomial equation $h_{1}^{2}(\omega)+h_{2}^{2}(\omega)=1$, where $h_{2}(\omega)=\sin \omega \gamma$. In a similar way to Case (a) we find the value of $\omega$ in view of $h_{1}(\omega)=\cos \omega \gamma$, we have

$$
\gamma_{2}^{(j)}=\frac{1}{\omega}\left[\arccos h_{1}(\omega)+2 j \pi\right], \quad j=0,1,2, \ldots
$$

Here $d_{1}^{2}(\omega)+d_{2}^{2}(\omega)=1$, which has at least one positive real root, thus the bifurcation point is defined as

$$
\gamma_{0}=\min \left\{\gamma_{1}^{(j)}, \gamma_{2}^{(j)}\right\}, \quad j=0,1,2, \ldots
$$

where $\gamma_{1}^{(j)}, \gamma_{2}^{(j)}$ are defined by (43) and (44). 

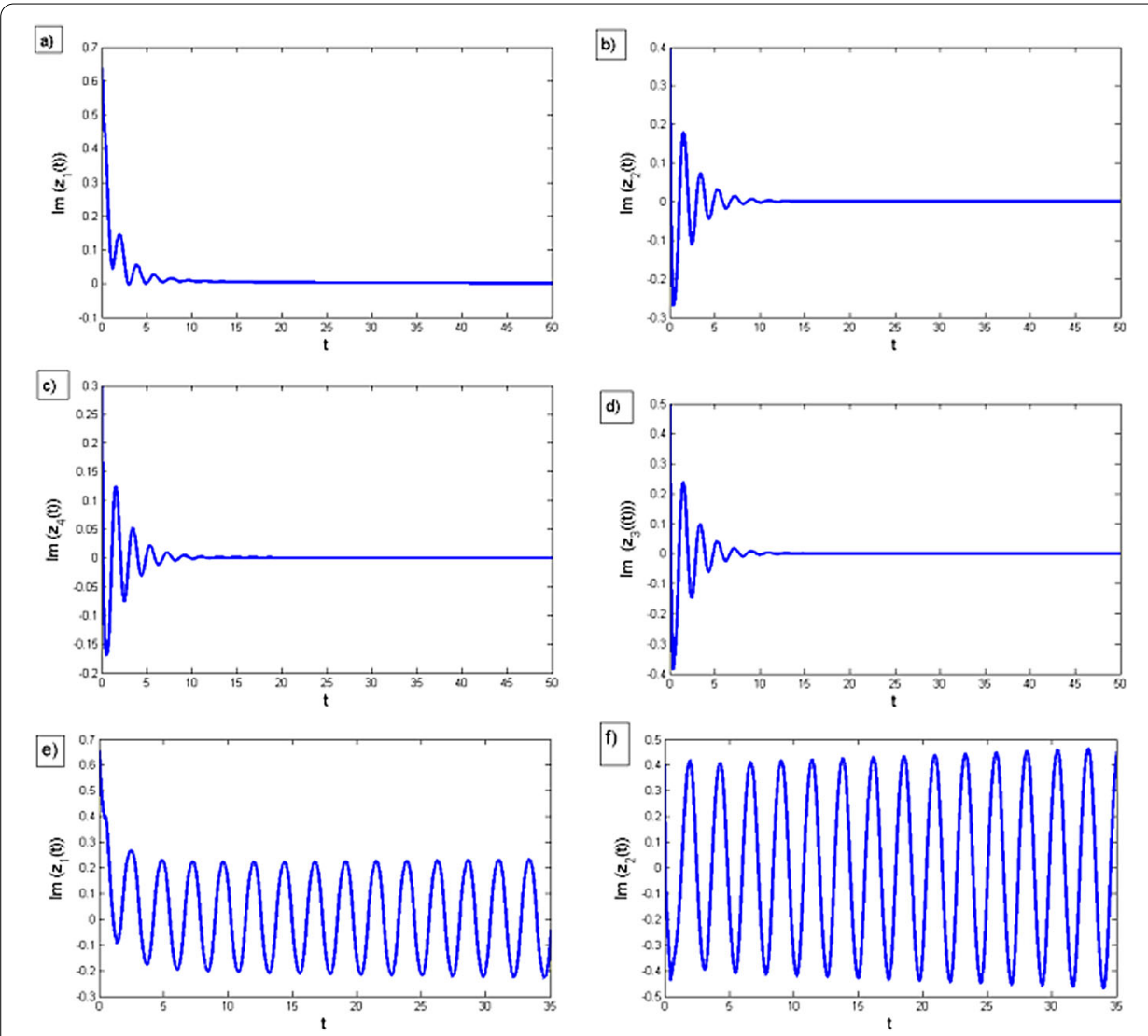

Figure 2 Curve of the imaginary parts of $z_{1}(t)$ in (a), $z_{2}(t)$ in (b), $z_{4}(t)$ in (c) and $z_{3}(t)$ in (d) and imaginary parts of $z_{1}(t)$ in (e) and $z_{2}(t)$ in (f) of fractional-order CVHNN with hub structure (50) with $\alpha=0.9, \gamma=0.31<\gamma_{0}=0.32$ and $\gamma=0.45>\gamma_{0}=0.32$, respectively.

Now, we analyze the stability of system (30) when $\gamma=0$, so the characteristic equation (40) becomes

$$
q(\beta)=\beta^{2}+C_{1} \beta+C_{2}+C_{3} \beta+C_{4}+C_{5}=0 .
$$

Then (46) can be written as

$$
\beta^{2}+\rho_{1} \beta+\rho_{2}=0
$$

where

$$
\rho_{1}=C_{1}+C_{3}, \quad \rho_{2}=C_{2}+C_{4}+C_{5} .
$$

It is defined as

$$
\chi_{1}=\rho_{1}, \quad \chi_{2}=\left|\begin{array}{cc}
\rho_{1} & 1 \\
\rho_{3} & \rho_{2}
\end{array}\right|, \quad \chi_{3}=\left|\begin{array}{ccc}
\rho_{1} & 1 & 0 \\
\rho_{3} & \rho_{2} & \rho_{1} \\
0 & \rho_{4} & \rho_{3}
\end{array}\right|, \quad \ldots, \quad \chi_{n}=\chi_{n-1} \rho_{n} .
$$

(A2) $\chi_{i}>0(i=1,2)$ 

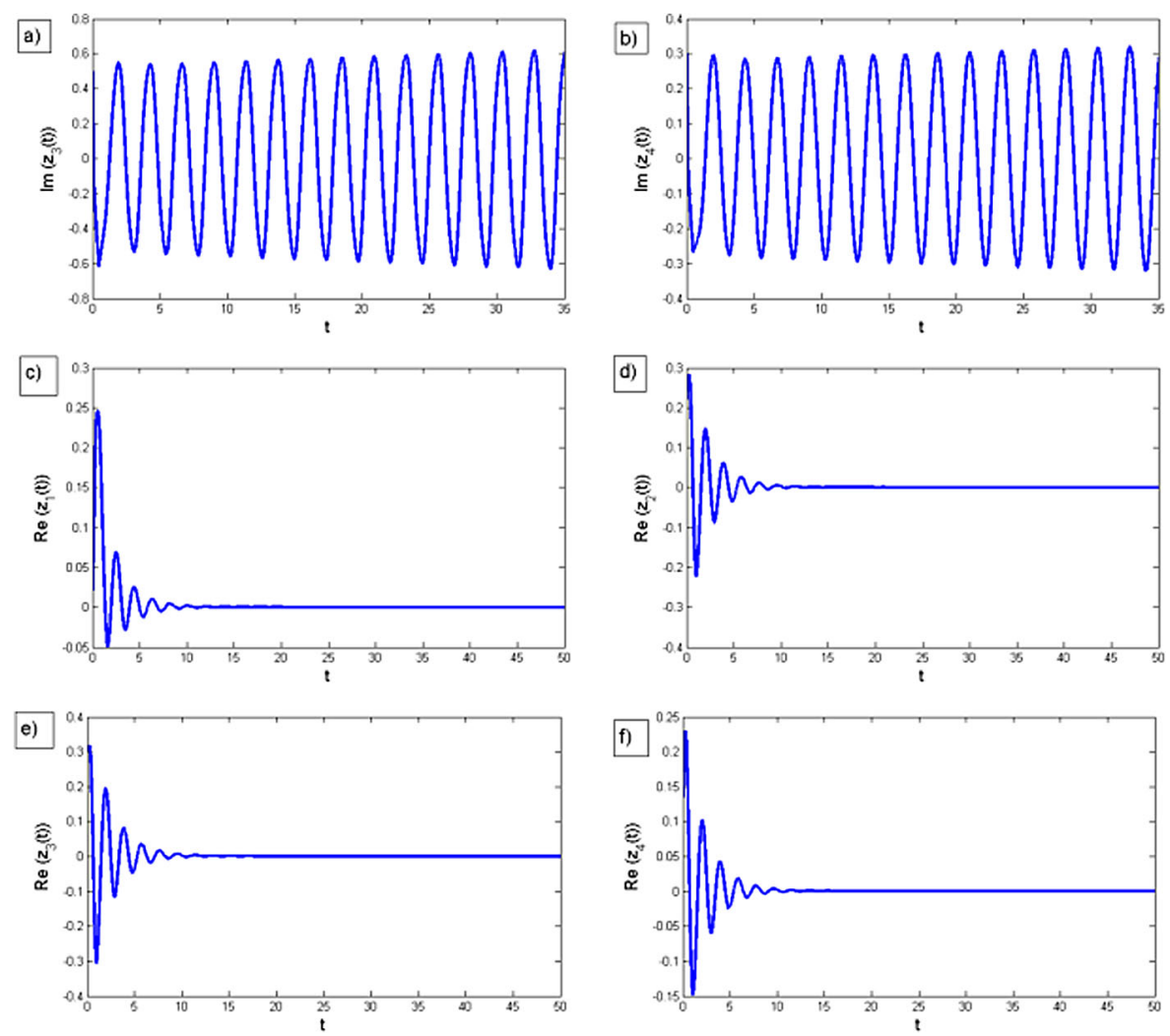

Figure 3 Curve of the imaginary parts of $z_{3}(t)$ in $(a), z_{4}(t)$ in (b) and real parts of $z_{1}(t)$ in $(c), z_{2}(t)$ in (d), $z_{3}(t)$ in (e) and $z_{4}(t)$ in (f) of fractional-order CVHNN with hub structure (50) with $\alpha=0.9$, $\gamma=0.45>\gamma_{0}=0.32$ and $\gamma=0.31<\gamma_{0}=0.32$, respectively.

Lemma 5 If $\chi_{1}>0$ and $\chi_{2}>0$, then the equilibrium point of the fractional-order CVHNNs with ring structured and time delay system (30) is stable when $\gamma=0$.

Now, considering the transversality condition of the occurrence for a Hopf bifurcation, it is given by

(A3) $\left.\operatorname{Re}\left[\frac{d s}{d \gamma}\right]\right|_{\left(\gamma=\gamma_{0}, \omega=\omega_{0}\right)} \neq 0$,

where $\omega_{0}$ and $\gamma_{0}$ are the critical frequency and the bifurcation point. Taking the derivative of $s$ with respect to $\gamma$ in (39), we have

$$
\frac{d s}{d \gamma}=\frac{X(s)}{Y(s)}
$$

coming by the same procedure as in the hub structure of earlier section, we have the condition for the Hopf bifurcation

$$
\left.\operatorname{Re}\left[\frac{d s}{d \gamma}\right]\right|_{\left(\gamma=\gamma_{0}, \omega=\omega_{0}\right)}=\frac{M_{1} N_{1}+M_{2} N_{2}}{M_{1}^{2}+M_{2}^{2}},
$$

where $N_{1}, N_{2}$ are the real and imaginary parts of $X(s)$ and $M_{1}, M_{2}$ are the real and imaginary parts of $Y(s)$. The terms in $M_{1}, M_{2}, N_{1}$ and $N_{2}$ have the same meaning as where 

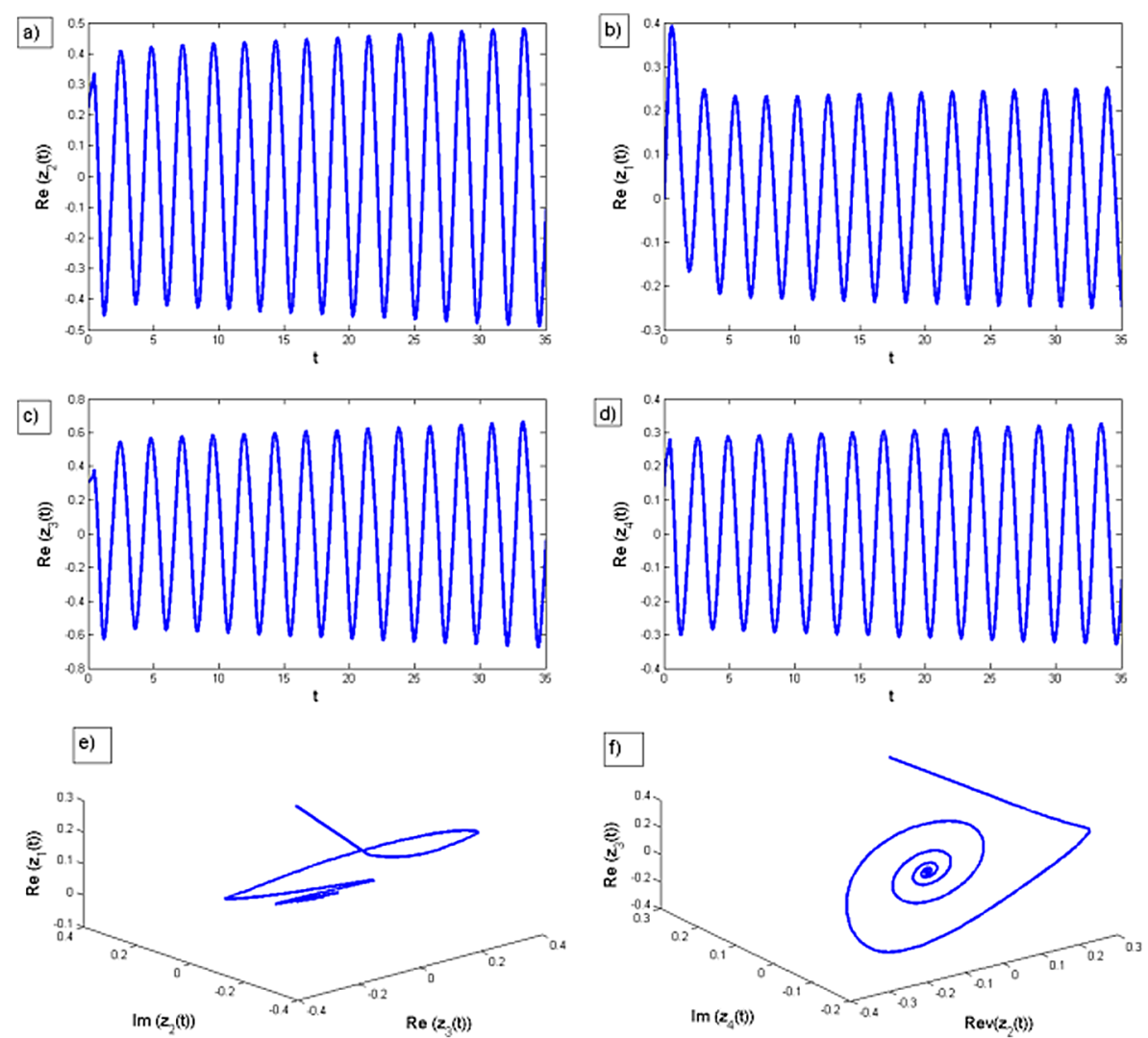

Figure 4 Curve of the real parts of $z_{2}(t)$ in (a), $z_{1}(t)$ in (b), $z_{3}(t)$ in (c) and $z_{4}(t)$ in (d), and (e), (f) are phase portraits of the real and imaginary parts of $z_{1}(t), z_{2}(t), z_{3}(t)$ and $z_{4}(t)$ of fractional-order CVHNN with hub structure (50) with $\alpha=0.9, \gamma=0.45>\gamma_{0}=0.32$ and $\gamma=0.31<\gamma_{0}=0.32$, respectively.

we mentioned $Y_{1}, Y_{2}, X_{1}$ and $X_{2}$ in Section 3.1. The above analysis leads to the following theorem.

Theorem 2 Assume that $(\mathbb{H} 1)-(\mathbb{H} 2)$ and $(\mathbb{A} 1)-(\mathbb{A} 3)$ are satisfied for fractional-order system (30), the following results hold:

(1) The zero equilibrium point is locally asymptotically stable for $\gamma \in\left[0, \gamma_{0}\right)$.

(2) The fractional-order system (30) exhibits a Hopf bifurcation at the origin when $\gamma=\gamma_{0}$, that is, the system (30) has a branch of periodic solutions bifurcating from the zero equilibrium point near $\gamma=\gamma_{0}$.

\section{Numerical examples}

In this section, numerical examples are given to verify the effectiveness of the analytical results. The simulation results are based on the Adams-Bashforth-Moulton predictorcorrector scheme for considered examples. The step length is taken in all the examples as $h=0.01$.

Example 1 In this example, the occurrence of Hopf bifurcation problem is investigated for the fractional-order CVHNNs with the hub structure and time delay system (7). Consider the following fractional-order CVHNNs (for arbitrary order $\alpha=0.9$ ) of four neurons with 


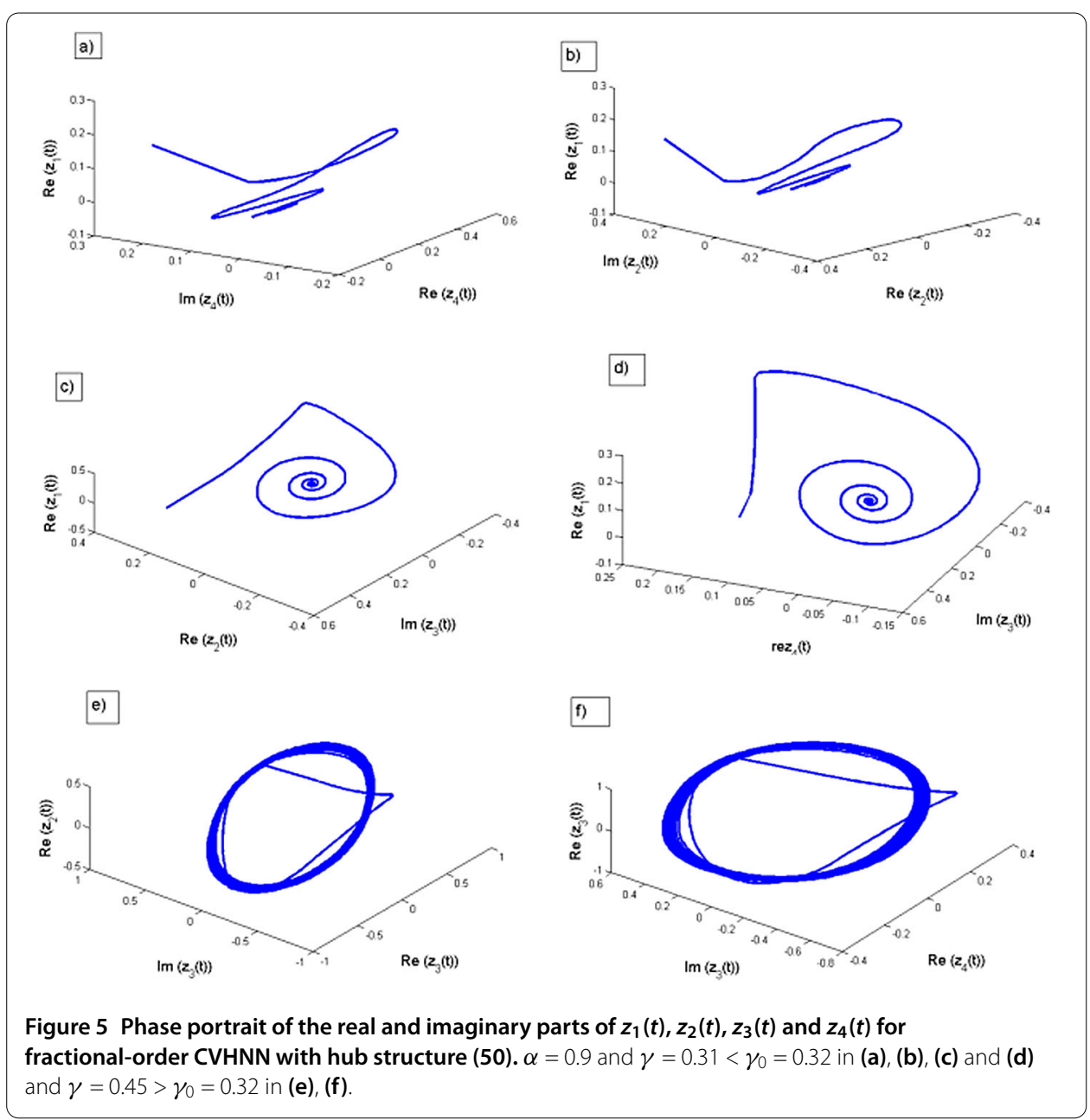

hub structure and time delay:

$$
\left\{\begin{aligned}
{ }_{0}^{c} D_{t}^{0.9} z_{1}(t)= & -3 z_{1}(t)+(-1+1.5 i) f_{1}\left(z_{1}(t)\right)+(-2.4+3.2 i) f_{2}\left(z_{2}(t)\right) \\
& +(3-0.3 i) f_{3}\left(z_{3}(t)\right)+(1.6-1.5 i) f_{4}\left(z_{4}(t)\right) \\
& +(2-i) f_{1}\left(z_{1}(t-\gamma)\right), \\
{ }_{0}^{c} D_{t}^{0.9} z_{2}(t)= & -2.8 z_{2}(t)+(2+0.2 i) f_{2}\left(z_{2}(t)\right)+(-2-2 i) f_{2}\left(z_{2}(t-\gamma)\right), \\
{ }_{0}^{c} D_{t}^{0.9} z_{3}(t)= & -2.8 z_{3}(t)+(2+0.2 i) f_{3}\left(z_{2}(t)\right)+(-2-2 i) f_{3}\left(z_{2}(t-\gamma)\right), \\
{ }_{0}^{c} D_{t}^{0.9} z_{4}(t)= & -2.8 z_{4}(t)+(2+0.2 i) f_{4}\left(z_{2}(t)\right)+(-2-2 i) f_{4}\left(z_{2}(t-\gamma)\right) .
\end{aligned}\right.
$$

Here the activation function is $f(z)=\tanh (z)$, it is obvious that system (50) admits a zero equilibrium point with the complex derivative $f^{\prime}(0)=1$. The considered fractional-order CVHNNs (50) is compared to (7), we have $c_{1}=3, c_{2}=c_{3}=c_{4}=2.8, b_{1}=2-i, b=-2-2 i$, $a_{11}=-1+1.5 i, a_{22}=a_{33}=a_{44}=2+0.2 i, a_{12}=-2.4+3.2 i, a_{13}=3-0.3 i$, and $a_{14}=1.6-1.5 i$, the initial values of the activation function are chosen as $z_{1}(0)=0.1+0.6 i, z_{2}(0)=0.2+$ $0.4 i, z_{3}(0)=0.3+0.5 i, z_{4}(0)=0.1+0.3 i$. We derive the critical frequency $\omega_{0}=0.42$ and the bifurcation point $\gamma_{0}=0.32$. Figure 1 shows the occurrence of a bifurcation point at $\gamma_{0}=0.32$ and the system is unstable when $\gamma=0.45>\gamma_{0}=0.32$. According to Theorem 1, 

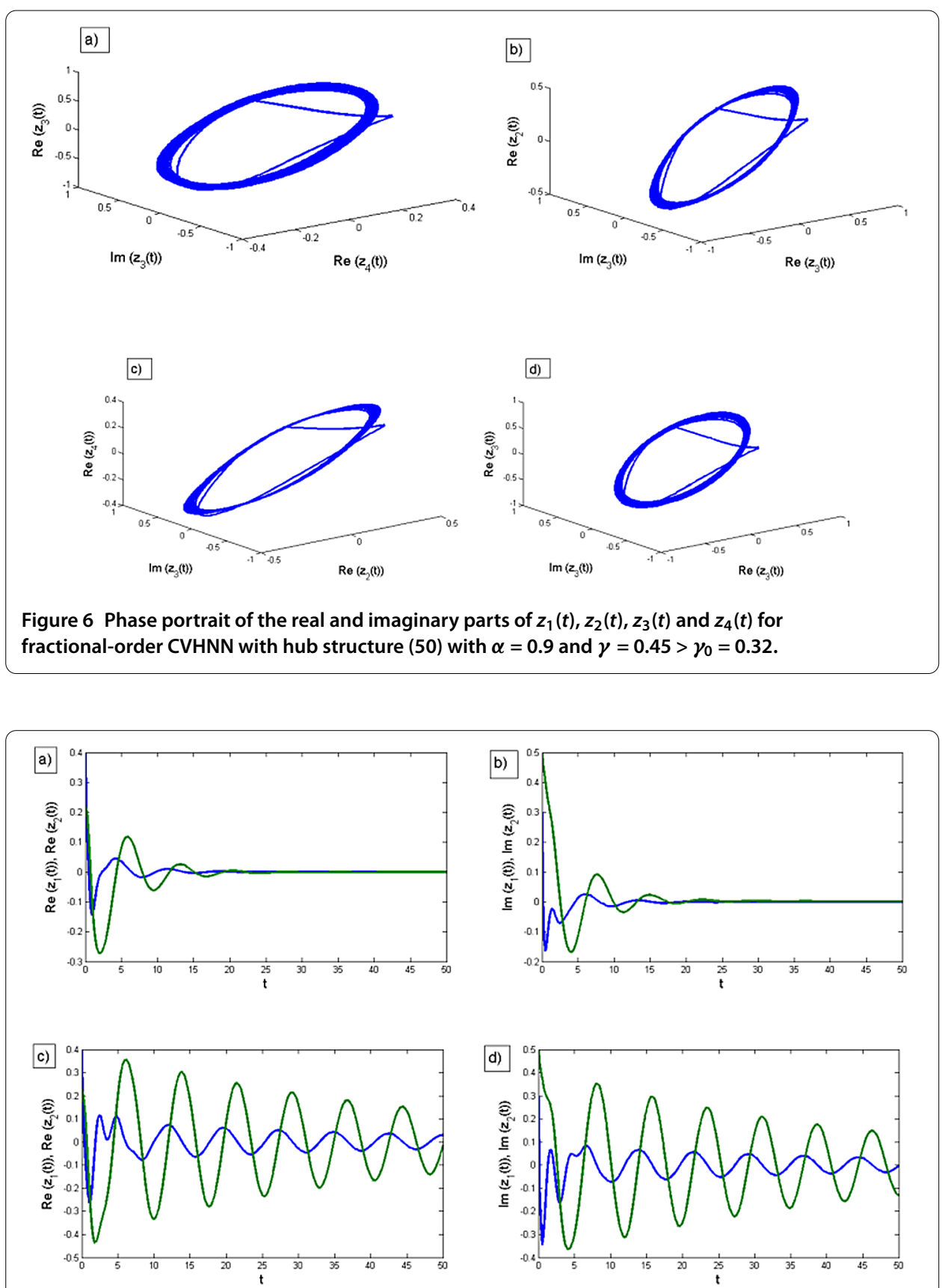

Figure 7 State trajectories of the real and imaginary parts of the fractional-order CVHNN with ring structure (51) with $\alpha=0.9, \gamma_{0}=0.32$ in (a), (b) and $\gamma=0.45>\gamma_{0}=0.3$ in (c), (d).

the zero equilibrium point $(0,0,0,0)$ is asymptotically stable when $\gamma=0.31<\gamma_{0}=0.32$, which is depicted in Figure 2. In Figure 3, we show that the curve for the imaginary parts of $z_{3}(t)$ and $z_{4}(t)$ are unstable for $\gamma=0.45>\gamma_{0}=0.32$ and real parts of $z_{1}, z_{2}, z_{3}, z_{4}$ are stable for $\gamma=0.31<\gamma_{0}=0.32$. In Figure 4 , we show the curve for the real parts is unstable for the system (50) for $\gamma=0.45>\gamma_{0}=0.32$ and a stability phase portrait is depicted in Figure 4 and Figure 5 , for $\gamma=0.31<\gamma_{0}=0.32$. Figure 6 illustrates the phase portrait of the instability for (50), when $\gamma=0.45>\gamma_{0}=0.32$. 

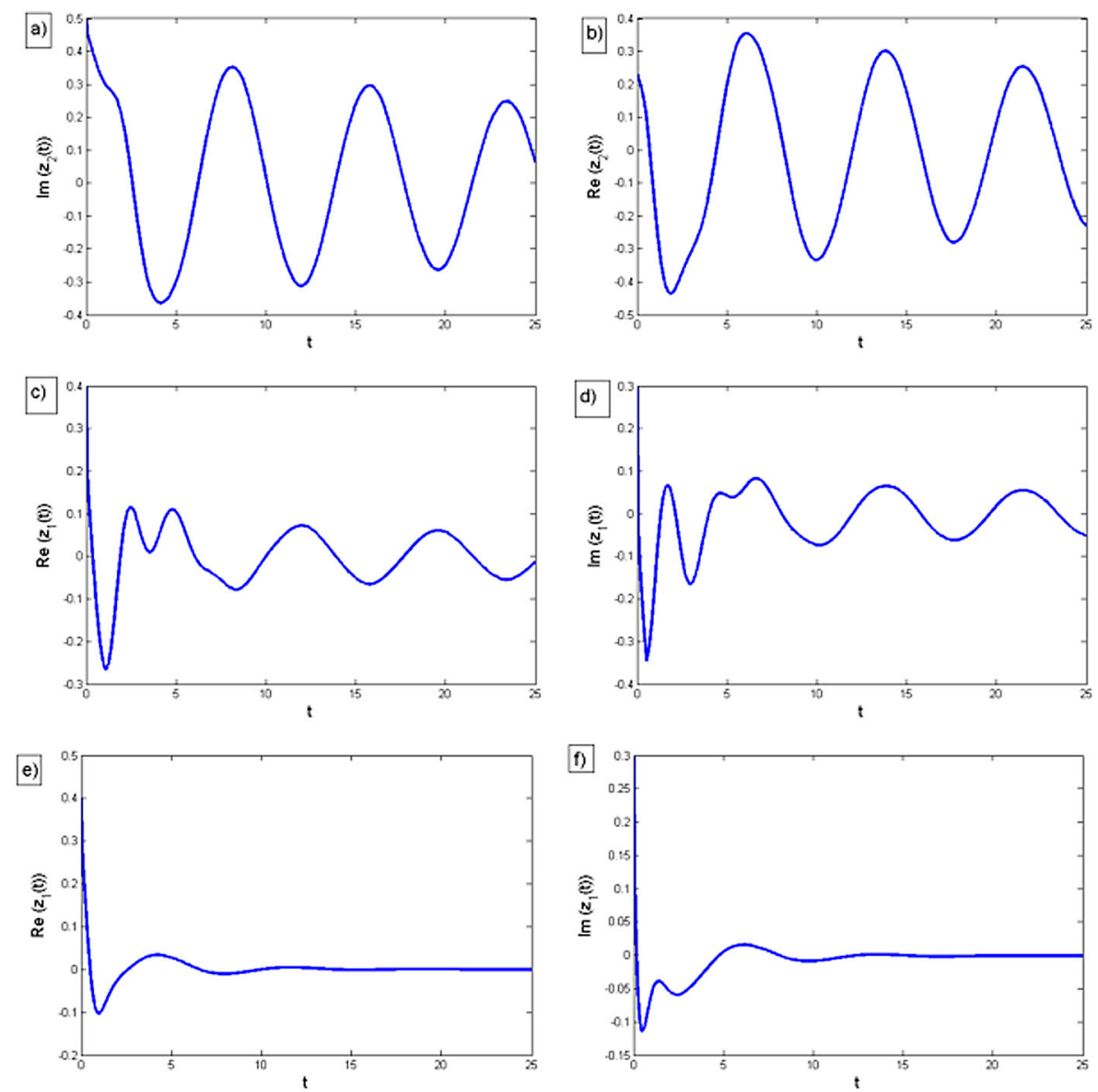

Figure 8 Curve of the real and imaginary part of $z_{2}(t), z_{1}(t)$ for fractional-order CVHNN with ring structure (51). $\alpha=0.9, \gamma=0.45>\gamma_{0}=0.3$ in (b), (a), (c), (d) and $\gamma=0.45>\gamma_{0}=0.3$ and real and imaginary parts of $z_{1}(t)$ with $\alpha=0.9, \gamma=0.2<\gamma_{0}=0.3$ in (e), (f)

Example 2 Consider the following fractional-order CVHNNs of two neurons with ring structure and time delay:

$$
\left\{\begin{aligned}
{ }_{0}^{c} D_{t}^{0.9} z_{1}(t)= & -c_{1} z_{1}(t)+a_{11} f_{1}\left(z_{1}(t)\right)+a_{12} f_{2}\left(z_{2}(t)\right)+b_{11} f_{1}\left(z_{1}(t-\gamma)\right) \\
& +b_{12} f_{2}\left(z_{2}(t-\gamma)\right), \\
{ }_{0}^{c} D_{t}^{0.9} z_{2}(t)= & -c_{2} z_{2}(t)+a_{22} f_{2}\left(z_{2}(t)\right)+a_{21} f_{1}\left(z_{1}(t)\right)+b_{11} f_{2}\left(z_{2}(t-\gamma)\right) \\
& +b_{21} f_{1}\left(z_{1}(t-\gamma)\right) .
\end{aligned}\right.
$$

The values for (51) are chosen as $c_{1}=0.2, c_{2}=-0.3, a_{11}=-0.3-0.4 i, a_{22}=-0.5-0.4 i$, $a_{12}=-0.6+0.2 i, a_{21}=0.5-0.3 i$, and $b_{11}=-1-1 i, b_{22}=-0.3+1 i, b_{12}=0.1+0.3 i, b_{21}=0.5-$ $0.3 i$. The activation functions are defined as $f_{1}\left(z_{1}(t)\right)=\tanh \left(z_{1}\right), f_{2}\left(z_{2}(t)\right)=\tanh \left(z_{2}\right)$ and the initial values are chosen as $z_{1}(0)=0.4+0.3 i, z_{2}(0)=0.2+0.5 i$. The critical frequency $\omega_{0}=$ 1.25 and the bifurcation point $\gamma_{0}=0.3$ is obtained in Maple by simple calculations. The occurrence of the bifurcation point $\gamma_{0}=0.3$ is shown in Figure 7 and also the system (51) is unstable for $\gamma=0.45>\gamma_{0}=0.3$. According to Theorem 2, the zero equilibrium point is locally asymptotically stable when $\gamma=0.2<\gamma_{0}=0.3$, which is illustrated in Figure 8 and 

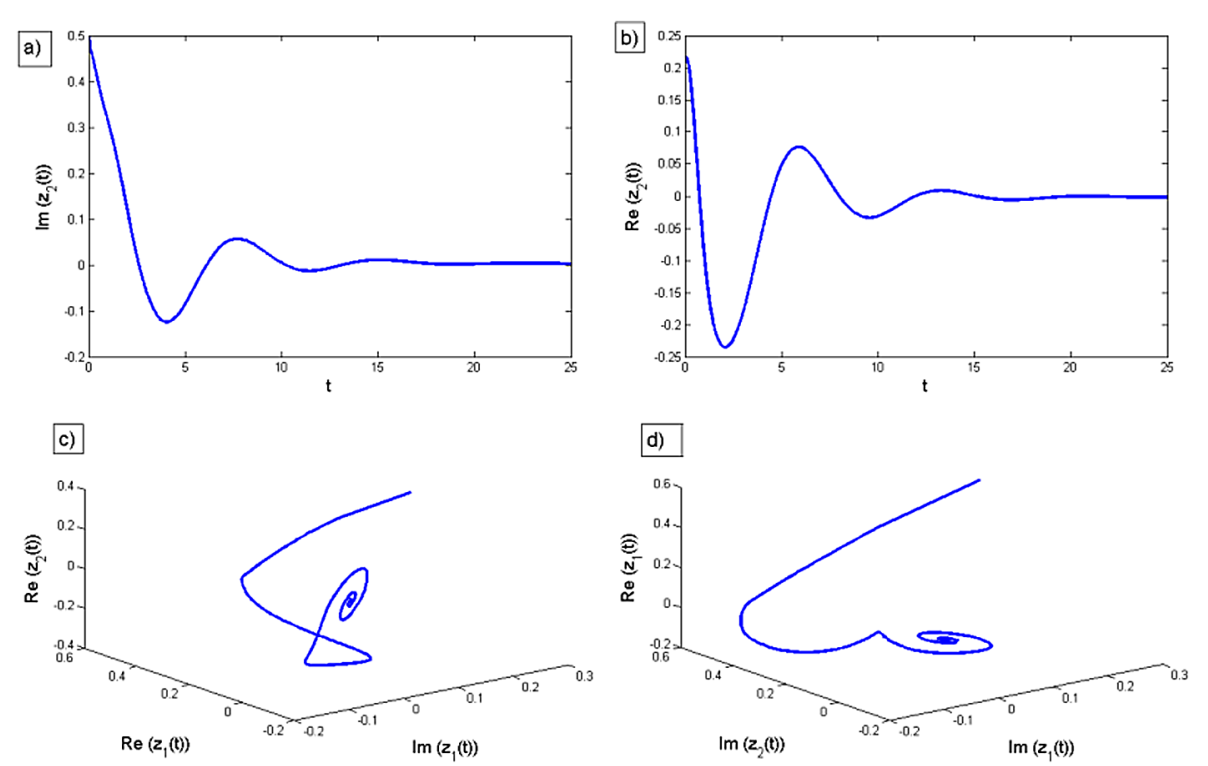

Figure 9 Curve of the real and imaginary part of $z_{2}(t)$ for fractional-order CVHNN with ring structure (51) with $\alpha=0.9$ and $\gamma=0.2<\gamma_{0}=0.3$ in (b), (a) and phase portrait of the real and imaginary parts of $z_{1}(t), z_{2}(t)$ with $\alpha=0.9$ and $\gamma=0.2<\gamma_{0}=0.3$ in (c), (d).
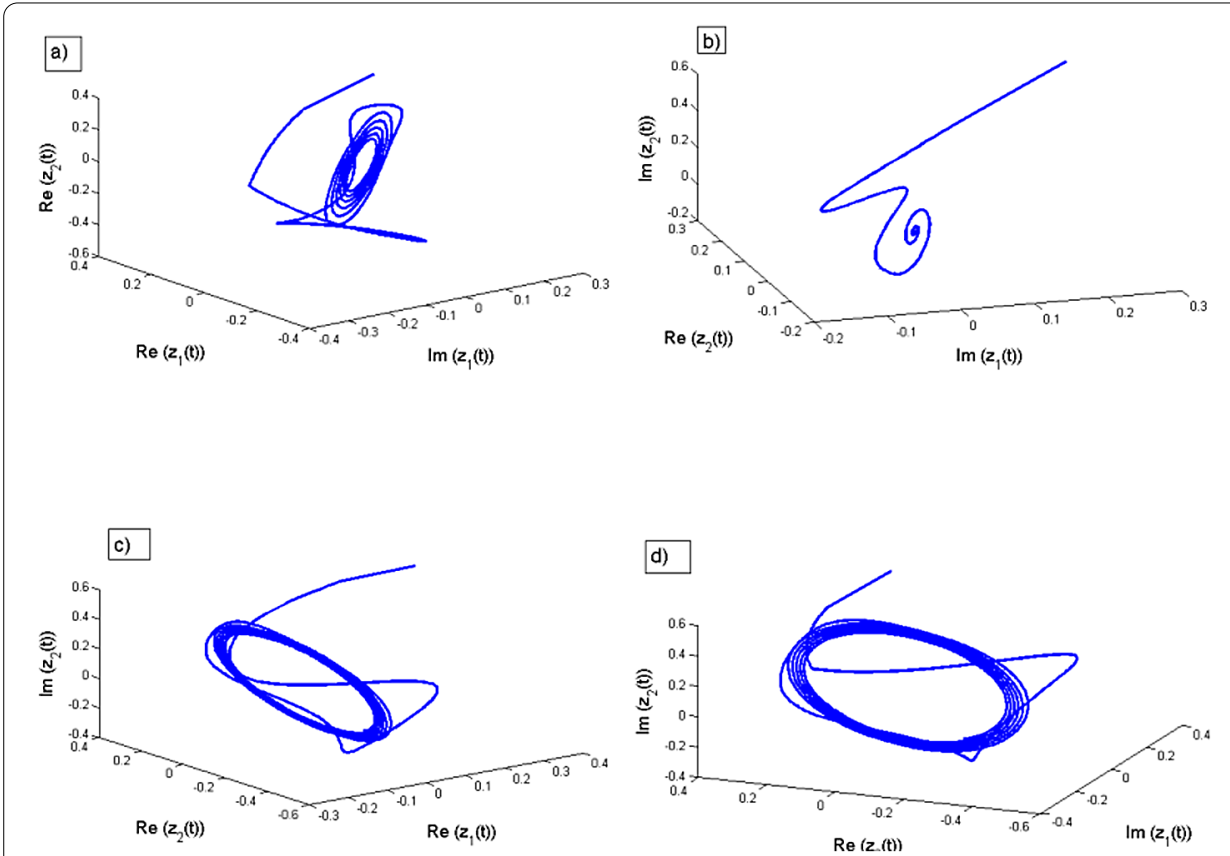

Figure 10 Phase portrait of the real and imaginary parts of $z_{1}(t), z_{2}(t)$ for a fractional-order CVHNN with ring structure (51) with $\alpha=0.9$ and $\gamma=0.2<\gamma_{0}=0.3$ in (b) and portrait of the real and imaginary parts of $z_{1}(t), z_{2}(t)$ with $\alpha=0.9$ and $\gamma=0.45>\gamma_{0}=0.3$ in (a),(c), (d).

Figure 9. The phase portraits of stable and unstable versions of system (51) are represented in Figure 10. 


\section{Conclusions}

In this paper, the class of fractional-order CVHNNs with hub and ring structured system is considered in a time delay sense. In this paper we use the Laplace transforms to the system of linearized equation to get the characteristic matrix for further stability analysis of the equilibrium which has been completely characterized. The critical frequency of the fractional-order $\alpha$ for which the corresponding bifurcation point may occur has been identified and the transversality condition is verified for the corresponding $\omega_{0}$ and $\gamma_{0}$. Moreover, the obtained conditions are simple, precise, effective and easy to verify. Numerical examples are given to verify the effectiveness of the theoretical results. Future work will focus on the analysis of stability and Hopf bifurcation of high-dimensional fractionalorder ring structure CVHNNs with multiple delays.

Acknowledgements

This work was supported by CSIR research project No.25(0237)/14/EMR-II.

Competing interests

The authors declare that they have no competing interests.

Authors' contributions

All authors contributed equally and significantly in writing this article. All authors read and approved the final manuscript.

\section{Author details}

${ }^{1}$ Department of Mathematics, Bharathiar University, Coimbatore, Tamilnadu 641 046, India. ${ }^{2}$ School of Mathematics and Research Center for Complex Systems and Network Sciences, Southeast University, Nanjing, 210096, China. ${ }^{3}$ Department of Mathematics, Faculty of Science, King Abdulaziz University, Jeddah, 21589, Saudi Arabia.

\section{Publisher's Note}

Springer Nature remains neutral with regard to jurisdictional claims in published maps and institutional affiliations.

Received: 8 March 2017 Accepted: 6 July 2017 Published online: 04 August 2017

\section{References}

1. Cochocki, A, Unbehauen, R: Neural Networks for Optimization and Signal Processing. Wiley, New York (1993)

2. Gopalsamy, K, He, X: Stability in asymmetric Hopfield nets with transmission delays. Phys. D: Nonlinear Phenom. 76, 344-358 (1994)

3. Huang, C, Cao, J, Xiao, M, Alsaedi, A, Hayat, T: Bifurcations in a delayed fractional complex-valued neural network. Appl. Math. Comput. 292, 210-227 (2017)

4. Hirose, A: Complex-Valued Neural Networks. Springer, Berlin (2006)

5. Kim, T, Adali, T: Fully complex multi layer perceptron network for nonlinear signal processing. J. VLSI Signal Process. Syst. Signal Image Video Technol. 32, 29-43 (2002)

6. Lin, S, Lu, C: Laplace transform for solving some families of fractional differential equations and its applications. Adv. Differ. Equ. 2013, 137 (2013)

7. Li, X, Rakkiyappan, R, Velmurugan, G: Dissipativity analysis of memristor-based complex-valued neural networks with time-varying delays. Inf. Sci. 294, 645-665 (2015)

8. Rakkiyappan, R, Cao, J, Velmurugan, G: Existence and uniform stability analysis of fractional-order complex-valued neural networks with time delays. IEEE Trans. Neural Netw. Learn. Syst. 26, 84-97 (2015)

9. Velmurugan, G, Rakkiyappan, R, Vembarasan, V, Cao, J, Alsaedi, A: Dissipativity and stability analysis of fractional-order complex-valued neural networks with time delay. Neural Netw. 86, 42-53 (2016)

10. Arik, S: Stability analysis of delayed neural networks. IEEE Trans. Circuits Syst. I 47, 1089-1092 (2000)

11. Wang, $H, Y u, Y$, Wen, G, Zhang, S: Stability analysis of fractional-order neural networks with time delay. Neural Process. Lett. 42, 479-500 (2015)

12. Hopfield, JJ, Tank, DW: Neural computation of decisions in optimization problems. Biol. Cybern. 52, 141-152 (1985)

13. Ding, Z, Shen, Y: Global dissipativity of fractional-order neural networks with time delays and discontinuous activations. Neurocomputing 196, 159-166 (2016)

14. Feng, C, Plamondon, R: On the stability analysis of delayed neural networks systems. Neural Netw. 14, 1181-1188 (2001)

15. Cao, J, Rakkiyappan, R, Maheswari, K, Chandrasekar, A: Exponential $H_{\infty}$ filtering analysis for discrete-time switched neural networks with random delays using sojourn probabilities. Sci. China, Technol. Sci. 59, 387-402 (2016)

16. Li, R, Cao, J: Stability analysis of reaction-diffusion uncertain memristive neural networks with time-varying delays and leakage term. Appl. Math. Comput. 278, 54-69 (2016)

17. Cao, J, Li, R: Fixed-time synchronization of delayed memristor-based recurrent neural networks. Sci. China Inf. Sci. 60 , $032201(2017)$

18. Chen, L, Qu, J, Chai, Y, Wu, R, Qi, G: Synchronization of a class of fractional-order chaotic neural networks. Entropy 15 , 3265-3276 (2013) 
19. Kriesel, D: A Brief Introduction on Neural Networks. Citeseer (2007)

20. Wang, Y, Li, T: Stability analysis of fractional-order nonlinear systems with delay. Math. Probl. Eng. 2014, Article ID 301235 (2014)

21. Xia, YS, Wang, J: A general projection neural network for solving monotone variational inequalities and related optimization problems. IEEE Trans. Neural Netw. 15, 318-328 (2004)

22. Huang, C, Cao, J: Active control strategy for synchronization and anti-synchronization of a fractional chaotic financial system. Phys. A, Stat. Mech. Appl. 473, 262-275 (2017)

23. Kaslik, E, Sivasundaram, S: Nonlinear dynamics and chaos in fractional-order neural networks. Neural Netw. 32 , 245-256 (2012)

24. Huang, C, Cao, J, Xiao, M: Hybrid control on bifurcation for a delayed fractional gene regulatory network. Chaos Solitons Fractals 87, 19-29 (2016)

25. Nitta, T: Orthogonality of decision boundaries in complex-valued neural networks. Neural Comput. 16, 73-97 (2004)

26. Rakkiyappan, R, Velmurugan, G, Cao, J: Finite-time stability analysis of fractional-order complex-valued memristor-based neural networks with time delays. Nonlinear Dyn. 78, 2823-2836 (2014)

27. Rakkiyappan, R, Sivaranjani, K, Velmurugan, G: Passivity and passification of memristor-based complex-valued recurrent neural networks with interval time-varying delays. Neurocomputing 144, 391-407 (2014)

28. Zhao, H: Global asymptotic stability of Hopfield neural network involving distributed delays. Neural Netw. 17, 47-53 (2014)

29. O'Kelly, ME: Routing traffic at hub facilities. Netw. Spat. Econ. 10, 173-191 (2010)

30. Liu, C, Li, C, Huang, T, Li, C: Stability of Hopfield neural networks with time delays and variable-time impulses. Neural Comput. Appl. 22, 195-202 (2013)

31. Song, C, Cao, J, Liu, Y: Robust consensus of fractional-order multi-agent systems with positive real uncertainty via second-order neighbors information. Neurocomputing 165, 293-299 (2015)

32. Song, C, Cao, J: Dynamics in fractional-order neural networks. Neurocomputing 142, 494-498 (2014)

33. Velmurugan, G, Rakkiyappan, R, Cao, J: Further analysis of global $\mu$-stability of complex-valued neural networks with unbounded time-varying delays. Neural Netw. 67, 14-27 (2015)

34. Chen, T: Global exponential stability of delayed Hopfield neural networks. Neural Netw. 14, 977-980 (2001)

35. Fang, Y, Kincaid, TG: Stability analysis of dynamical neural networks. IEEE Trans. Neural Netw. 7, 996-1006 (1996)

36. Liao, XF, Chen, G, Sanchez, EN: LMI-based approach for asymptotically stability analysis of delayed neural networks. IEEE Trans. Circuits Syst. I 49, 1033-1039 (2002)

37. Yu, W, Cao, J: Stability and Hopf bifurcation analysis on a four-neuron BAM neural network with time delays. Phys. Lett. A 351, 64-78 (2006)

38. Huang, C, Cao, J, Ma, Z: Delay-induced bifurcation in a tri-neuron fractional neural network. Int. J. Inf. Syst. Sci. 47, 3668-3677 (2016)

39. Xiao, M, Zheng, WX, Jiang, G, Cao, J: Undamped oscillations generated by Hopf bifurcations in fractional-order recurrent neural networks with Caputo derivative. IEEE Trans. Neural Netw. Learn. Syst. 12, 3201-3214 (2015)

40. Zhang, J, Jin, X: Global stability analysis in delayed Hopfield neural network models. Neural Netw. 13, 745-753 (2000)

41. Cao, J, Xiao, M: Stability and Hopf bifurcation in a simplified BAM neural network with two time delays. IEEE Trans. Neural Netw. 18, 416-430 (2007)

42. $\mathrm{Hu}, \mathrm{H}, \mathrm{Huang}, \mathrm{L}$ : Stability and Hopf bifurcation analysis on a ring of four neurons with delays. Appl. Math. Comput. 213, 587-599 (2009)

43. Li, X, Wei, J: On the zeros of a fourth degree exponential polynomial with applications to a neural network model with delays. Chaos Solitons Fractals 26, 519-526 (2005)

44. Wei, J, Yuan, Y: Synchronized Hopf bifurcation analysis in a neural network model with delays. J. Math. Anal. Appl. 312 205-229 (2005)

45. Xu, W, Hayat, T, Cao, J, Xiao, M: Hopf bifurcation control for a fluid flow model of Internet congestion control systems via state feedback. IMA J. Math. Control Inf. 33, 69-93 (2016)

46. Huang, C, Meng, Y, Cao, J, Alsaedi, A, Alsaadi, FE: New bifurcation results for fractional BAM neural network with leakage delay. Chaos Solitons Fractals 100, 31-44 (2017)

47. Rakkiyappan, R, Velmurugan, G, Cao, J: Stability analysis of fractional-order complex-valued neural networks with time delays. Chaos Solitons Fractals 78, 297-316 (2015)

\section{Submit your manuscript to a SpringerOpen ${ }^{\circ}$ journal and benefit from:}

- Convenient online submission

- Rigorous peer review

- Open access: articles freely available online

- High visibility within the field

- Retaining the copyright to your article

Submit your next manuscript at $>$ springeropen.com 